\title{
Kinetic Monte Carlo simulation of the effective diffusivity in grain boundary networks
}

\author{
Jie Deng ${ }^{\mathrm{a}}$, Dane Morgan ${ }^{\mathrm{a}, \mathrm{b}}$, Izabela Szlufarska ${ }^{\mathrm{a}, \mathrm{b}, *}$ \\ ${ }^{a}$ Department of Materials Science and Engineering, University of Wisconsin-Madison, Madison, WI 53706 \\ ${ }^{b}$ Department of Engineering Physics, University of Wisconsin-Madison, Madison, WI 53706
}

\begin{abstract}
The effective diffusivity in grain boundary networks of polycrystalline materials is evaluated using a kinetic Monte Carlo model. This model connects the atomic hopping processes with the coarse-grained diffusion so that the macroscopic simulations can be conducted without the need to resolve atomic details. The effects of various properties of the grain boundary network on the effective diffusivity have been examined, including grain size, two- vs. three-dimensional networks, and distribution of grain boundary diffusivities. It is shown that the effective diffusivity does not depend on the grain size when grain boundary diffusion is the dominant diffusion mechanism in a polycrystalline sample. We find that the behavior of the effective diffusivity is qualitatively the same for two- and three-dimensional models, except that the percolation threshold and the critical exponents need to be changed accordingly when using empirical functions to characterize the effective diffusivity. In addition, we find that the effective diffusivity exhibits large fluctuations due to its dependence on the grain boundary distributions, and therefore the details of the materials microstructure can significantly impact the effective diffusivity in a specific finite-size sample. Finally, we check the applicable range of the effective medium theory and discuss the effects of modeling different grain boundary types with varying diffusivities using just two vs. many types. The grain boundary diffusion program (GBDiff) developed in this study is available under open source licensing as part of the MAterials Simulation Toolkit (MAST) and can be obtained from https://pypi.python.org/pypi/MAST/1.1.0.
\end{abstract}

Keywords: Grain boundary diffusion, Effective diffusivity, Kinetic Monte Carlo

\section{Introduction}

Grain boundary (GB) diffusion plays an important role in various processes occurring in polycrystalline materials such as grain growth, sintering, recrystallization, Coble creep and diffusioninduced GB migration [1, 2]. Since GBs generally provide high diffusivity paths and GB diffusion is one of the dominant transport mechanisms in materials, understanding the mechanism of GB diffusion and its dependence on GB structure is of significant interest. The first macroscopic GB diffusion model was developed by Fisher [3]. This model treats a single GB as a highly diffusive thin slab embedded in a semi-infinite body in such a way that the longer dimension of the slab is perpendicular to the surface where diffusion source exists. Solving this model under the fixed

\footnotetext{
${ }^{*}$ Corresponding author. Tel: +1 608-265-5878; Fax: +1 608-262-8353.

Email address: szlufarska@wisc.edu (Izabela Szlufarska)
} 
concentration boundary condition provides the diffusant's concentration profile along the GB, from which the GB diffusivity can be then determined. The penetration profile of diffusant can also be used to characterize the diffusion kinetics. One of the most widely used classification of diffusion kinetics is Harrison's classification, which introduces three regimes called type A, B and C [1, 4]. In type A regime, the bulk diffusion length is larger than the spacing between GBs. In type B regime, the bulk diffusion length is smaller than the GB spacing and GBs can be regarded as isolated. In type $\mathrm{C}$ regime, the bulk diffusion length is smaller than the GB width so that diffusion can be considered as taking place primarily within GBs with negligible sidewise leakage into the bulk [1]. Later on, Fisher's model was extended to account for different types of boundary conditions (e.g., constant or instantaneous source) and different GB configurations (e.g., single or parallel GBs in a semi-infinite or a finite domain) [5-11]. These models provided many insights into the GB diffusion processes along an isolated GB or along multiple GBs with the same diffusivity in an idealized geometry. Nevertheless, application of these models to GB networks with complex connectivity and GB-dependent diffusivities is not straightforward.

In real materials, GB diffusion is sensitive to the GB atomic structure. The structure dependence of GB diffusion was first measured by Turnbull and Hoffman [12, 13], where it was shown that the diffusion along the [001] tilt GBs in Ag is anisotropic and it depends on the misorientation between two grains. Since then, the misorientation dependence of GB diffusion has been found in many other systems. It is generally found that the diffusivity along high angle GBs is higher than along low angle or coincidence site lattice (CSL) GBs [14-17]. The structure dependence of GB diffusion arises from the atomic nature of diffusion. Specifically, GB diffusion generally depends on formation energies and migration energies of point defects and these properties strongly depend on the specific arrangements of atoms in the GB [18]. Because of this structure dependence, the diffusivities in individual GBs may vary by several orders of magnitude under typical testing conditions, which makes it challenging to describe the macroscopic diffusivity when mediated by GB diffusion in polycrystalline samples. In such cases, the effective diffusivity is one of the most important quantities used to characterize the macroscopic GB diffusion. The effective diffusivity is defined in such a way that the macroscopic diffusion through a heterogeneous GB network (where individual GBs have a wide range of diffusivities) is equivalent to the diffusion through a homogeneous system with a single value of the effective diffusivity. As the effective diffusivity is often correlated with various physical properties of structural materials related to GB diffusion, such as intergranular fracture, corrosion, and cavitation [19-23], understanding the dependence of the effective diffusivity on the details of the GB network is critical for design of materials with desirable properties.

One should note that the effective diffusivity through polycrystalline materials is a complex function of different properties of the GB network such as the GB distribution and the GB connectivity. Early investigations on the effective diffusivity were focused on developments of phenomenological diffusion models in the Harrison type-A kinetics regime [1,24-30]. These models account for the bulk diffusion and GB diffusion in different grain patterns (e.g., square grains with the same size surrounded by GB region uniformly distributed in the domain), but they generally consider only one GB type and simplified GB geometries, and therefore ignore the influence of GB variety and varied GB connectivity on the effective diffusivity. Here, we focus on the effective diffusivity in Harrison's type $\mathrm{C}$ kinetic regime where GB diffusion is much faster than bulk diffusion. One material science problem relevant to this regime may be the diffusion of $\mathrm{Ag}$ through polycrystalline silicon carbide ( $\mathrm{SiC})[31,32]$, where it has been suggested that the diffusivity of $\mathrm{Ag}$ in $\mathrm{SiC}$ GBs is several 
orders of magnitude larger than that in $\mathrm{SiC}$ bulk over a wide-range of temperature. In the type $\mathrm{C}$ kinetic regime, one can assume that the diffusion occurs along GBs only and that the properties of the GB network play a key role in determining the effective diffusivity. Recently, Chen and Schuh [33] provided new insights into the effective diffusivity in a two-dimensional (2D) honeycomb GB network. The authors investigated the role of a diffusivity contrast, defined as the ratio of the diffusivities along high-diffusivity and low-diffusivity GBs. The GB networks were categorized into high-contrast systems and low-contrast systems, where the diffusivity contrast is larger and smaller than $10^{4}$, respectively. It was shown that in high-contrast systems, the effective diffusivity is governed by the development of a percolating path of high-diffusivity GBs. In low-contrast systems, the effective diffusivity is less sensitive to the topology of the GB network and it can be described using composite averaging schemes.

The aim of our paper is twofold. First, we develop a kinetic Monte Carlo (kMC) model to calculate the effective diffusivity in static heterogeneous GB networks. This model is applicable for the case of impurity diffusion when the impurity has a strong tendency to segregate to GBs. In contrast to earlier kMC models that obtained the diffusivity in a single GB based on the jump rates of point defects [34-37], our model focuses on the long-range diffusion along GBs and determines the effective diffusivity by taking individual GB diffusivities as an input. Our model provides a connection between the coarse-grained motion of the diffusant and the atomic level processes underlying diffusion. This connection is validated numerically and it allows us to ignore the atomic details of GB diffusion in kMC simulations. Second, we extend the work of Chen and Schuh [33] to further account for the dependence of the effective diffusivity on various properties of the GB network. Instead of using a 2D honeycomb lattice, we use a three-dimensional (3D) Voronoi diagram to represent the GB network with non-uniform distributions of grain sizes and GB areas. Two or more types of GBs are assumed to be randomly distributed in the simulation domain. In a system with two types of GBs, we demonstrate the dependence of the effective diffusivity on diffusivity contrast, fraction of each GB type, GB distribution, and the average grain size. We also use our numerical models to test the validity of two ways of modeling the effective diffusivity, namely the effective medium theory (EMT) and the percolation theory. Finally, we discuss the effects of considering more than two types of GBs on the effective diffusivity.

\section{Kinetic Monte Carlo model}

In order to capture GB diffusion in 3D GB networks, we mesh each GB plane using the software Neper [38], which applies a widely-utilized bottom-up approach to generate mesh in Voronoi tessellation vertices, edges (GB junctions) and faces (GBs), successively. A typical 3D GB network with mesh in each GB is shown in Fig. 1a. For the sake of computational efficiency, our kMC model does not resolve the atomic hopping processes. Instead, a diffusing particle is assumed to perform a coarse-grained hop, i.e., it moves from one mesh node to another along an element edge instantaneously in each kMC step. Therefore the current model captures the motion of a particle on diffusive time scales and microscopic length scales, which is different from classic atomistic models where hops occur on the atomic scale. With the assumption of coarse-grained hop, the kMC calculation of the effective diffusivity can be carried out as follows.

A Voronoi diagram [38] with a specified number of grains is first generated in a 3D cubic domain to represent the GB network. In the Voronoi construction, grain centers are randomly distributed. Then each GB plane is meshed by triangular elements. Each GB is then randomly assigned to be one of two or more types, where each type has a different diffusivity. The diffusivity in a GB 
junction is assumed to be the same as the highest diffusivity in all GBs that connect to that GB junction. Then the diffusivity along each triangular element edge is set to be the diffusivity in the GB plane or GB junction it belongs to. Initially, a particle is located at a randomly selected mesh node, and it is allowed to diffuse in the domain via hops along element edges (i.e., from one mesh node to another). Periodic boundary conditions are applied in all spatial directions. In each kMC step, the particle chooses to move along a given element edge $i$ randomly based on the rate $\Gamma_{i}=\frac{D_{i}}{l_{i}^{2}}$, where $D_{i}$ and $l_{i}$ are the diffusivity and the length of element edge $i$, respectively. After each step, the total diffusion time $\tau$ and the total displacement $d$ (displacement between particle's initial position and its current position) are recorded. After a sufficiently long time (long enough to obtain converged results), the effective diffusivity can be calculated as $D_{\text {eff }}=\frac{d^{2}}{6 \tau}$. This procedure is repeated for $5 \times 10^{4}$ polycrystalline samples, and the reported effective diffusivities are the average values. We also develop a model to calculate the effective diffusivity on a 2D Voronoi GB network. The simulation method in 2D GB networks is similar to that in 3D GB networks. The main difference is that in 2D, each Voronoi tessellation edge represents a GB so that no mesh is needed and a particle hops between tessellation vertices. In addition, in 2D the effective diffusivity is calculated by $D_{\text {eff }}=\frac{d^{2}}{4 \tau}$.

We note that our model relies on the assumption that a diffusing particle performs a coarsegrained hop (it moves from one mesh node to another) instead of atomic-level hops between nodes. However, we propose that this coarse-grained hopping yields almost the same effective diffusivity as an atomically resolved hopping simulation, since the rates of hopping between mesh nodes are chosen to give the correct average time to move such a distance. In order to validate this proposition and to connect the coarse-grained hop and atomic-level hops, we perform kMC simulations in a $2 \mathrm{D}$ rectangular domain with a square mesh. In this case, the entire $2 \mathrm{D}$ simulation domain corresponds to a $3 \mathrm{D}$ element edge, where the domain size $L$ in the horizontal direction is the edge length, and the mesh size $h$ is the atomic hopping distance. In simulations, two parallel surfaces perpendicular to the horizontal direction, i.e., left and right surfaces, are free surfaces, and periodic boundary conditions are applied along other directions that are perpendicular to the horizontal direction so that the size of the simulation domain along these directions is irrelevant to the results. In each simulation, a particle is initially placed at the left boundary, and it is allowed to diffuse by hopping from one lattice point to another. The particle can move in four directions at all lattice points except at the left boundary where it can move only in three directions (up, down, right). The probability of choosing a given hopping direction is proportional to the hopping rate in that direction, and the hopping rate is given by $\Gamma_{0}=\frac{D_{0}}{h^{2}}$, where $D_{0}$ is the atomic diffusion coefficient. Using this hopping rate, we measure the time $\tau$ one particle requires to move from the left to the right boundary, and then determine the diffusivity for the coarse-grained hop in this domain using the Einstein's equation $D=\frac{L^{2}}{2 \tau}$.

We calculate the diffusivity $D$ for different values of parameters $L, h$ and $D_{0}$, and we find that in all cases, $D$ for the coarse-grained hop is equal to the diffusion coefficient $D_{0}$ for the atomic hopping, that is, $D=\frac{L^{2}}{2 \tau}=D_{0}$ within numerical error of the simulation. We confirmed the validity of this equality in a 3D domain with a cubic mesh. In 3D, we also consider the diffusion between two parallel free surfaces perpendicular to the horizontal direction, and apply periodic boundary conditions to all other surfaces. These results also demonstrate that $D=D_{0}$ within the numerical error regardless of the domain size, the hopping distance and the dimensionality of the simulation domain. This relationship connects the atomic diffusion coefficient $D_{0}$ with the diffusivity $D$ of the coarse-grained hop, where the particle moves from one mesh node to another. It implies that 
the macroscopic diffusion in a GB network can be captured by coarse-grained hops of a particle without resolving the details of atomic hopping processes.

There are several significant additional assumptions of the model that should be mentioned here. First, the only diffusion mechanism allowed in our model is the GB diffusion. As mentioned before, most previous models for the effective diffusivity combines GB diffusion with other diffusion mechanisms (e.g., bulk or dislocation pipe diffusion) [1, 24-30]. Our simulations are focused on long-range diffusion processes in a macroscopic domain where the GB diffusion is the dominant mechanism. The second assumption is that GBs with different characters are randomly distributed. It is known that GB types in experimental samples are correlated and subject to crystallographic constraints [39-41]. However, the only effect of GB correlation is a few percent shift in the percolation threshold, while the critical exponents in the percolation theory are not affected by the correlation [33]. Because of that, we ignore the GB correlation in this work. The reader should keep in mind that the percolation threshold in actual GB networks may be slightly different from the one predicted by our model. If needed, correlations in GB types can be added to our model framework relatively easily in future work. Finally, in our model the diffusion coefficient at the GB junction and the GB are taken as identical. While the diffusion along GB junctions is generally different from that in GBs, their difference depends on temperature and GB structure, and the relative magnitude of the diffusivity in GB junctions and GBs is still in debate [42, 43]. As such, the diffusion coefficients along GB junctions are treated as equal to diffusion coefficients along GBs in the current model.

The GB diffusion model described above has been programmed into a tool called GBDiff. GBDiff is available under open source licensing as part of the MAterials Simulation Package [44] and can be obtained from https://pypi.python.org/pypi/MAST. The version of GBDiff used for this paper is GBDiff version 1.0 in MAST version 1.2.0. When using the GBDiff code please cite the present paper and any additional references given in the MAST citation file, which is generated automatically when MAST is run. GBDiff can also be used as on online application through the Materials Hub (materialhub.org(http://materialhub.org)) [45]. Please follow the citation guidelines given for this tool on the MaterialsHub.

\section{Results for systems with two types of GBs}

The kMC model introduced in Section 2 is first applied to calculate the effective diffusivity $D_{\text {eff }}$ in a system containing two types of GBs: high-energy GBs (HEGBs) with diffusivity $D_{\mathrm{H}}$ and low energy GBs (LEGBs) with diffusivity $D_{\mathrm{L}}$. These GB types are randomly distributed in the GB network. In order to determine the effect of system dimensionality on diffusion through GB networks, we consider two different GB topologies based on 2D and 3D Voronoi diagrams.

The GB network in our systems is characterized by the diffusivity contrast $\frac{D_{\mathrm{H}}}{D_{\mathrm{L}}}$, the fraction $f_{\mathrm{H}}$ of HEGBs , the distribution of GB types and the average grain size. In addition to these properties, the connectivity of HEGBs can also play an important role in determining $D_{\text {eff }}$. The influence of the HEGB connectivity on macroscopic material properties has been shown in Ref. [1923, 33]. The effect of the HEGB connectivity is particularly apparent in systems with two types of GBs, as considered in our study, because HEGBs can form percolating paths for the diffusant and profoundly affect $D_{\text {eff }}[33]$. Therefore, before we calculate $D_{\text {eff }}$ in our systems, we first evaluate the percolation threshold of the Voronoi GB networks. Following the method in Ref. [46], we investigate the percolation paths between two parallel free surfaces and apply periodic boundary 
conditions to all other surfaces. The final result of the percolation threshold is independent of which free surface we choose and instead, it depends on the connectivity of HEGBs in the system. Note that the boundary conditions used here for calculating the percolation threshold are different from the periodic boundary conditions applied when calculating the effective diffusivity. According to Ref. [46], a HEGB is called percolating if it is directly connected to the selected surface (the right boundary in our study) or if one of its nearest HEGB neighbors is percolating. With this criterion, we are able to identify the number of percolating HEGBs in the GB network, and then the fraction of percolating HEGBs can be obtained. Ideally, in a system with an infinite number of GBs, the fraction of percolating HEGBs will change from zero to unity when the fraction of HEGBs $f_{\mathrm{H}}$ is equal to the percolation threshold $f_{\mathrm{p}}$. In a system with finite number of GBs, when $f_{\mathrm{H}}$ approaches $f_{\mathrm{p}}$, the fraction of percolating HEGBs will change from zero to unity gradually but rapidly, and we can approximate $f_{\mathrm{p}}$ from the change in the fraction of percolation HEGBs.

Since the fraction of percolating HEGBs is strongly dependent on the specific GB distribution for a finite system, in order to obtain statistically relevant results, we carry out a large number of simulations (typically $10^{4}$ ) for each value of $f_{\mathrm{H}}$. The average value of the fraction of percolating HEGBs are reported. As shown in Fig. 1b, the percolation threshold $f_{\mathrm{p}}$ is $0.67 \pm 0.01$ and $0.18 \pm 0.01$ for the $2 \mathrm{D}$ and $3 \mathrm{D}$ Voronoi GB networks, respectively. Here, $f_{\mathrm{p}}$ is determined by the value of $f_{\mathrm{H}}$ where the fraction of percolating GBs is 0.5 , and the uncertainty is approximated by the range of $f_{\mathrm{H}}$ where the fraction of percolation GBs varies from $\frac{1}{3}$ to $\frac{2}{3}$. The percolation threshold in $2 \mathrm{D}$ is consistent with the results of Monte Carlo simulations reported in Refs. [47, 48], where the authors found $f_{\mathrm{p}}=0.66693$ in the $2 \mathrm{D}$ Voronoi network. In addition, $f_{p}$ can be used to estimate the effective coordination number $\mathrm{Z}$ according to the formula [46] $Z f_{p}=\frac{d}{d-1}$, where $d$ is the dimensionality. Using the values of $f_{p}$ and $d$, we find that $Z$ is about 3.0 and 8.3 for $2 \mathrm{D}$ and 3D Voronoi diagrams, respectively.

Before we evaluate the dependence of the effective diffusivity on the properties of the 3D GB network, we check the dependence of the effective diffusivity on the mesh density. Fig. 1c shows the effective diffusivity in a 3D GB network with $2 \times 10^{3}$ grains as a function of mesh density with different GB network properties. It can be seen that within the range of mesh density considered here, the effective diffusivity is not very sensitive to the mesh density. In the following, we carry out $\mathrm{kMC}$ diffusion simulations in a 3D Voronoi GB network with $4 \times 10^{3}$ grains where the average number of elements in one GB is approximately 9 . In these simulations, $D_{\mathrm{L}}$ is fixed at a constant value (which is 1 in our study), and we vary $D_{\mathrm{H}}$ to change the diffusivity contrast $\frac{D_{\mathrm{H}}}{D_{\mathrm{L}}}$. As a result, the observed trends in the dependence of diffusion on $\frac{D_{\mathrm{H}}}{D_{\mathrm{L}}}$ are equivalent to the trends in the dependence on $D_{\mathrm{H}}$. Moreover, in the following figures, $D_{\text {eff }}$ is normalized by $D_{\mathrm{H}}$ so that the features of $D_{\text {eff }}$ observed in our study are not affected by the diffusivity unit. This normalization does not impact the dependence of $D_{\text {eff }}$ on the properties of the GB network.

\subsection{Dependence of $D_{\text {eff }}$ on the diffusivity contrast $D_{H} / D_{L}$}

Figure 2 shows $\frac{D_{\text {eff }}}{D_{\mathrm{H}}}$ as a function of $\frac{D_{\mathrm{H}}}{D_{\mathrm{L}}}$ for different values of $f_{\mathrm{H}}$. In this figure, the solid lines and the dashed lines correspond to the cases when $f_{\mathrm{H}}<f_{\mathrm{p}}$ and $f_{\mathrm{H}}>f_{\mathrm{p}}$, respectively, where the percolation threshold $f_{\mathrm{p}}=0.16$ (see Fig. 1c). We find that the dependence of $D_{\text {eff }}$ on $\frac{D_{\mathrm{H}}}{D_{\mathrm{L}}}$ when $f_{\mathrm{H}}<f_{\mathrm{p}}$ is different from that when $f_{\mathrm{H}}>f_{\mathrm{p}}$. In particular, when $f_{\mathrm{H}}<f_{\mathrm{p}}$, $D_{\text {eff }}$ is nearly independent of $D_{\mathrm{H}}$ so that $\frac{D_{\text {eff }}}{D_{\mathrm{H}}}$ is proportional to $\left(\frac{D_{\mathrm{H}}}{D_{\mathrm{L}}}\right)^{p}$ with the power coefficient $p \approx-1$. This result means that when the fraction of HEGBs is lower than the percolation threshold, the effective 
diffusivity is governed primarily by the diffusivity along LEGBs. On the other hand, for the case of $f_{\mathrm{H}}>f_{\mathrm{p}}$ and when $\frac{D_{\mathrm{H}}}{D_{\mathrm{L}}}$ increases, the dependence of $D_{\text {eff }}$ on $D_{\mathrm{H}}$ changes from a power law with exponent $p(-1<p<0)$ to a linear function $(p \approx 0)$. The transition from a power law to a linear function depends on $f_{\mathrm{H}}$ so that with higher $f_{\mathrm{H}}, D_{\text {eff }}$ becomes linearly dependent on $D_{\mathrm{H}}$ at smaller values of $\frac{D_{\mathrm{H}}}{D_{\mathrm{L}}}$. When the fraction of HEGBs exceeds the percolation threshold, $D_{\text {eff }}$ is dominated by $D_{\mathrm{H}}$ and this dominance becomes more apparent as $\frac{D_{\mathrm{H}}}{D_{\mathrm{L}}}$ increases. This behavior is expected because above the percolation threshold, the diffusion through the polycrystalline samples is accomplished mainly by the diffusion along HEGBs. As $\frac{D_{\mathrm{H}}}{D_{\mathrm{L}}}$ increases, the probability of diffusing via LEGBs pathways is further decreased, and eventually $D_{\text {eff }}$ becomes a linear function of $D_{\mathrm{H}}$. To summarize the trends in Fig. 2, $f_{\mathrm{H}}$ determines how $D_{\text {eff }}$ depends on $\frac{D_{\mathrm{H}}}{D_{\mathrm{L}}}$. When $f_{H}<f_{p}, D_{\text {eff }}$ is governed by $D_{\mathrm{L}}$ and is nearly independent of $\frac{D_{\mathrm{H}}}{D_{\mathrm{L}}}$. When $f_{H}>f_{p}, D_{\text {eff }}$ is approximately a linear function of $\frac{D_{\mathrm{H}}}{D_{\mathrm{L}}}$.

\subsection{Dependence of $D_{\text {eff }}$ on the fraction of $H E G B f_{\mathrm{H}}$}

While $f_{\mathrm{H}}$ determines the dependence of $D_{\text {eff }}$ on $\frac{D_{\mathrm{H}}}{D_{\mathrm{L}}}$ as illustrated in Fig. 2, the ratio $\frac{D_{\mathrm{H}}}{D_{\mathrm{L}}}$ affects how $D_{\text {eff }}$ depends on $f_{\mathrm{H}}$. As shown in Ref. [33] for systems with a uniform grain size and uniform GB length, the dependence of $D_{\text {eff }}$ on $f_{\mathrm{H}}$ follows different rules in high- and low-diffusivity contrast systems. Here we evaluate $D_{\text {eff }}$ as a function of $f_{\mathrm{H}}$ for different values of $\frac{D_{\mathrm{H}}}{D_{\mathrm{L}}}$ in the GB network with non-uniform grain size and GB length distributions. The results of our simulations are shown in Fig. 3a. In the same figure, we also show predictions of the effective medium theory (EMT) [50], which is a widely used analytical model for describing macroscopic transport properties in heterogeneous media. As shown in Fig. 3a, predictions of EMT agree well with the results of kMC simulations for small values of $\frac{D_{\mathrm{H}}}{D_{\mathrm{L}}}$. However, deviations between two approaches are observed as $\frac{D_{\mathrm{H}}}{D_{\mathrm{L}}}$ increases. This trend is consistent with the conclusions from Ref. [33] that EMT fails to predict $D_{\text {eff }}$ in high contrast systems. The discrepancy between EMT and kMC is due to the fact that EMT averages the diffusivities of all GBs, but it does not take into account the GB connectivity. As some HEGBs may form high-diffusivity paths and the diffusion prefers to occur along these paths, the overall diffusivity is higher than that estimated by the EMT.

Two other interesting features are found in Fig. 3a. First, the deviation of EMT from kMC in this study is larger than the one reported in Ref. [33]. Specifically, the authors of Ref. [33] found that EMT works reasonably well when $\frac{D_{\mathrm{H}}}{D_{\mathrm{L}}}<10^{4}$, while we find that EMT provides a poor approximation of the simulation data when $\frac{D_{\mathrm{H}}}{D_{\mathrm{L}}}>10^{2}$. The difference in the range of applicability of EMT arises from the difference in the GB connectivity in the two studies. More specifically, the percolation threshold in the GB network considered in our study is lower than that in Ref. [33]. Consequently, in the present study, the formation of long-range connectivity of HEGBs is easier, leading to a failure of the EMT approximation at smaller values of $\frac{D_{\mathrm{H}}}{D_{\mathrm{L}}}$. Secondly, we find that the largest deviation between EMT and kMC occurs when $f_{\mathrm{H}} \approx f_{\mathrm{p}}$. To illustrate this deviation, in Fig. $3 \mathrm{~b}$ we plot the ratio of $D_{\text {eff }}$ obtained from $\mathrm{kMC}$ to that predicted by EMT. It is clear from this plot that when $\left|f_{\mathrm{H}}-f_{\mathrm{p}}\right|>0.2$, EMT works quite well even at large values of $\frac{D_{\mathrm{H}}}{D_{\mathrm{L}}}$. On the other hand, when $\left|f_{\mathrm{H}}-f_{\mathrm{p}}\right|<0.2$, EMT and kMC predictions differ, and the difference reaches the maximum at $f_{\mathrm{H}}=f_{\mathrm{p}}$. We conclude that EMT provides a reasonable approximation for the effective diffusivity not only for low contrast systems, but also for high contrast systems when the absolute difference 
between $f_{\mathrm{H}}$ and $f_{\mathrm{p}}$ is large. This extension of the applicability of EMT makes it possible to use this theory to predict $D_{\text {eff }}$ in a wider range of GB structures than previously assumed.

As demonstrated in Figs. 3a and 3b, when $\frac{D_{\mathrm{H}}}{D_{\mathrm{L}}}$ increases, the percolating network of HEGBs begins to dominate diffusion and it is expected that $\frac{D_{\text {eff }}}{D_{\mathrm{H}}}$ in high contrast systems can be approximated by the percolation theory. Based on the percolation theory described in detail in Ref. [50, 51], $D_{\text {eff }}$ should exhibit a power-law dependence $D_{\text {eff }} \propto\left(f_{\mathrm{H}}-f_{\mathrm{p}}\right)^{t}$ when $f_{\mathrm{H}}>f_{\mathrm{p}}$ and $D_{\text {eff }} \propto\left(f_{\mathrm{p}}-f_{\mathrm{H}}\right)^{-s}$ when $f_{\mathrm{H}}<f_{\mathrm{p}}$, where $t$ and $s$ are critical exponents. Figures $3 \mathrm{c}$ and $3 \mathrm{~d}$ illustrate the dependence of $D_{\text {eff }}$ on $\left|f_{\mathrm{p}}-f_{\mathrm{H}}\right|$ for the cases of $f_{\mathrm{H}}>f_{\mathrm{p}}$ and $f_{\mathrm{H}}<f_{\mathrm{p}}$, respectively, where the solid lines represent the curve fitting using the above power-law functions. One can see that as $\frac{D_{\mathrm{H}}}{D_{\mathrm{L}}}$ increases, the relation between $D_{\text {eff }}$ and $\left|f_{\mathrm{p}}-f_{\mathrm{H}}\right|$ approaches the power laws shown above. In particular, when $\frac{D_{\mathrm{H}}}{D_{\mathrm{L}}}>10^{5}$, all the $\frac{D_{\text {eff }}}{D_{\mathrm{H}}}$ vs. $\left|f_{\mathrm{p}}-f_{\mathrm{H}}\right|$ functions can be well described by above power-law functions with exponents $t=1.30 \pm 0.05$ and $s=1.22 \pm 0.05$. This feature has also been observed in Ref. [33], but the critical exponents in that study were different from the ones reported here, presumably due to the difference in the type of GB network (e.g., 2D honeycomb lattice and 3D Voronoi diagram). The values of $t$ and $s$ found in our study are consistent with most of previous studies [50-58] on percolation systems, where the critical exponents are in the range of $1-2$.

While EMT and the percolation theory can predict $D_{\text {eff }}$ when $\frac{D_{\mathrm{H}}}{D_{\mathrm{L}}}$ is low and high, respectively, a generalized effective medium (GEM) equation [59-61] has been often used to predict $D_{\text {eff }}$ in the entire range of $\frac{D_{\mathrm{H}}}{D_{\mathrm{L}}}$ values. This equation has been previously applied in Ref. [33] to fit $D_{\text {eff }}$ in a 2D honeycomb GB lattice. The GEM equation has the following form

$$
\left(1-f_{\mathrm{H}}\right) \frac{D_{\mathrm{L}}^{1 / s}-D_{\mathrm{eff}}^{1 / s}}{D_{\mathrm{L}}^{1 / s}+\left(f_{\mathrm{p}}^{-1}-1\right) D_{\mathrm{eff}}^{1 / s}}+f_{\mathrm{H}} \frac{D_{\mathrm{H}}^{1 / t}-D_{\mathrm{eff}}^{1 / t}}{D_{\mathrm{H}}^{1 / t}+\left(f_{\mathrm{p}}^{-1}-1\right) D_{\mathrm{eff}}^{1 / t}}=0,
$$

where $f_{\mathrm{H}}, f_{\mathrm{p}}, D_{\mathrm{H}}, D_{\mathrm{L}}, s$ and $t$ have the same meanings as before. As shown earlier, in the case of our GB networks, $f_{\mathrm{p}}=0.18, t=1.30$, and $s=1.22$. By substituting these parameters into Eq. (1) we obtain the GEM prediction of $D_{\text {eff. }}$ The comparison of GEM and kMC calculation of $D_{\text {eff }}$ is illustrated in Fig. 3a, which shows that two methods match quite well. This result indicates that $D_{\text {eff }}$ in a system with two types of GBs can be approximated by the GEM equation with the values for the percolation threshold and the critical exponents being appropriate for the given GB network. A combined analysis of Figs. 2 and 3 demonstrates that the effects of $f_{\mathrm{H}}$ and $\frac{D_{\mathrm{H}}}{D_{\mathrm{L}}}$ on $D_{\text {eff }}$ are coupled to each other and are strongly dependent on the corresponding GB network.

\subsection{Statistical distribution of $D_{\mathrm{eff}}$}

As discussed in Section 2, the effective diffusivities reported so far in Figs. 2-3 are the averages over $5 \times 10^{4}$ samples. The standard error (as estimated by the standard deviation divided by the mean) of the reported average $D_{\text {eff }}$ is around $1 \%$. We note that the standard error decreases with both the number of samples, as to be expected, and the size of the system, as increasing the latter provides better averaging over a range of local environments (see Fig. 4a for an example). These results indicate that in order to have a more accurate measurement of the effective diffusivity in an experiment, we may either increase the system size or have more samples, which is as expected.

In addition to the standard error in the mean $D_{\text {eff }}$, the fluctuation in $D_{\text {eff }}$ among $5 \times 10^{4}$ samples is also of interested because it provides the spectrum of $D_{\text {eff }}$ that can be measured in a given sample and it reveals the dependence of $D_{\text {eff }}$ on the details of the microstructure. $D_{\text {eff }}$ exhibits fluctuations 
due to the statistical nature of GB distributions. Here we characterize the fluctuation in $D_{\text {eff }}$ using the coefficient of variation, $c_{v}=\frac{\sigma}{\mu}$, where $\mu=\left\langle D_{\text {eff }}\right\rangle$ is the average $D_{\text {eff }}$ and $\sigma$ is the standard

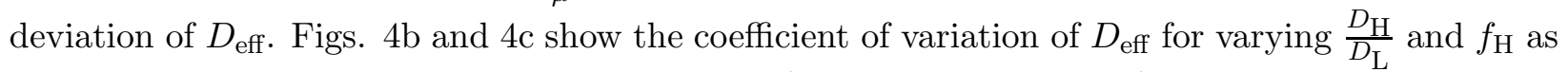
a function of the system size. One can see that $c_{v}$ (i.e., fluctuation in $D_{\text {eff }}$ ) generally decreases as the system size increases and the behavior of $c_{v}$ is not very sensitive to $\frac{D_{\mathrm{H}}}{D_{\mathrm{L}}}$ and $f_{\mathrm{H}}$. Moreover, it is found that for the system size studied in this work (i.e., $10^{2} \sim 10^{4}$ grains in $3 \mathrm{D}$ domain), $c_{v}$ is larger than 0.8 , which demonstrates that the fluctuation in $D_{\text {eff }}$ can be significant even if we have more than $10^{3}$ grains in a system. The spread of the $D_{\text {eff }}$ indicates that although the macroscopic diffusion can be characterized by the mean value of $D_{\text {eff }}$, the details of the microstructure should be taken into account when trying to predict $D_{\text {eff }}$ if diffusion will occur through a specific polycrystalline sample.

\subsection{Dependence of $D_{\text {eff }}$ on the model dimensionality}

In order to examine the dependence of $D_{\text {eff }}$ on the dimensionality, we evaluate $D_{\text {eff }}$ on a $2 \mathrm{D}$ Voronoi GB network with $10^{4}$ grains. The simulation conditions in the 2D Voronoi GB network are the same as that in the 3D Voronoi GB network. The corresponding figures for 2D Voronoi GB networks are shown in the supplemental document of the present paper.

We first analyze the dependence of $D_{\text {eff }}$ on $\frac{D_{\mathrm{H}}}{D_{\mathrm{L}}}$ for different values of $f_{\mathrm{H}}$, and we find that the trends are similar to those found in 3D Voronoi GB network (shown in Fig. 2). Specifically, $D_{\text {eff }}$ in $2 \mathrm{D}$ Voronoi GB network is nearly independent of $\frac{D_{\mathrm{H}}}{D_{\mathrm{L}}}$ when $f_{\mathrm{H}}$ is smaller than the percolation threshold $f_{\mathrm{p}}$, which for $2 \mathrm{D}$ Voronoi diagram is equal to 0.67 . When $f_{\mathrm{H}}>f_{\mathrm{p}}, D_{\text {eff }}$ becomes a linear function of $\frac{D_{\mathrm{H}}}{D_{\mathrm{L}}}$. We also find that the dependence of $D_{\text {eff }}$ on $f_{\mathrm{H}}$ follows the same trend as that shown in Fig. 3. Specifically, the EMT is accurate for low-contrast systems and the power-law functions in the percolation theory provide a good model for $D_{\text {eff }}$ in high-contrast systems. Significant deviation of EMT predictions from kMC results are observed mainly when $f_{\mathrm{H}}$ and $f_{\mathrm{p}}$ are close in values. In addition, we find that, similarly as in 3D Voronoi GB network, the GEM theory (Eq. (1) with exponents $t=1.6$ and $s=1.3$ ) can be used to approximate $D_{\text {eff }}$ in 2D Voronoi GB network. The agreement between the kMC and GEM methods again demonstrates that with the appropriate percolation threshold and critical exponents, the GEM theory can predict $D_{\text {eff }}$ with a reasonable accuracy. We also analyze the coefficient of variation of $D_{\text {eff }}$. We find that, similarly as in a 3D GB network, the coefficient of variation of $D_{\text {eff }}$ in a 2D Voronoi GB network decreases as the system size increases, and it does not strongly depend on $f_{\mathrm{H}}$ and $\frac{D_{\mathrm{H}}}{D_{\mathrm{L}}}$. Moreover, the fluctuation of $D_{\text {eff }}$ in a 2D Voronoi GB network can also be significant for a system containing at lease up to $10^{4}$ grains.

In summary, the dependence of $D_{\text {eff }}$ on $\frac{D_{\mathrm{H}}}{D_{\mathrm{L}}}$ and $f_{\mathrm{H}}$ as well as the fluctuation of $D_{\text {eff }}$ are nearly independent of the dimensionality of the simulation domain. The observed trends are similar in 2D and in 3D Voronoi GB networks. The main difference between 2D and 3D lies in the percolation threshold and the critical exponents in the percolation theory.

\subsection{Grain size effect of $D_{\text {eff }}$}

Grain size has been previously shown to affect diffusion through a polycrystalline sample, in cases where both GB diffusion and bulk diffusion are active [30]. As the grain size decreases, the fraction of GB region increases, which allows more diffusion to be conducted along GBs rather than through the crystalline grains. Since the GB diffusion is faster than the bulk diffusion, $D_{\text {eff }}$ 
increases as the grain size decreases. Here, we are interested in the kinetic regime where bulk diffusion through crystalline grains is negligible as compared to GB diffusion. It is interesting to ask whether the previously observed grain size effect will be present in this regime as well. We check three types of conditions: (1) the grain size is uniform and all GBs have the same character; (2) the grain size is uniform and GBs have two different characters; (3) the grain size is non-uniform and GBs have two different characters.

The grain size effect in the first case has been in fact already analyzed in Section 2, where the GB lattice is a square mesh in a 2D domain. We found that as the grain size decreases, the number of hopping steps across a certain distance increases. Meanwhile, the time spent in each hopping step decreases because the grain size decreases and so does the hopping distance. As a consequence, the total time over which one particle diffuses across a certain distance is independent of the grain size, and in this scenario $D_{\text {eff }}$ does not depend on the grain size. $D_{\text {eff }}$ in this case can actually be derived analytically following the steps shown in Ref. [62]. Specifically, the average square of the displacement of the diffusant after $n$ jumps can be expressed as $\left\langle d^{2}\right\rangle=n l^{2}$, where $l$ is the distance in each jump. Since the total diffusion time $\tau$ is $\tau=n \Delta t$ (where $\Delta t$ is the time spent in each jump),

it can be derived that $D_{\text {eff }}=\frac{\left\langle d^{2}\right\rangle}{4 \tau}=\frac{l^{2}}{4 \Delta t}$, so the effective diffusivity is equal to the diffusivity in each GB, and therefore it is independent of the grain size.

The next step (case (2)) is to consider two types of GBs (HEGBs and LEGBs) with different diffusivities $\left(D_{\mathrm{H}}\right.$ and $\left.D_{\mathrm{L}}\right)$. Analytical derivation of $D_{\text {eff }}$ in a system with two types of GBs is not straightforward since $D_{\text {eff }}$ depends on the GB connectivity as shown in Figure 3, so we focus on numerical calculation of $D_{\text {eff }}$ here. We calculate the numerical values of $D_{\text {eff }}$ as a function of grain size, where the GB lattice is a square mesh and two types of GBs with diffusivity contrast $\frac{D_{\mathrm{H}}}{D_{\mathrm{L}}}=10^{3}$ are randomly distributed. The results show that $D_{\text {eff }}$ still does not exhibit any grain size dependence within our error bars. We further evaluate $D_{\text {eff }}$ in a 2D Voronoi diagram with a non-uniform grain size and two types of GBs (case (3)), which again shows that $D_{\text {eff }}$ does not depend on the grain size.

Above results suggest that when GB diffusion is the only active mechanism of transport, $D_{\text {eff }}$ is independent of the grain size under quite general conditions. This finding is based on the assumption that the properties of the GB network (e.g., GB diffusivity, fraction of each type of GB) do not depend on the grain size. Therefore, if the grain size effect of $D_{\text {eff }}$ is observed in experiments on systems in the Type $\mathrm{C}$ regime, it will likely be induced by the relation between the grain size and the aforementioned properties of GBs. For instance, the authors of Ref. [63] have shown that the diffusion of hydrogen in pure nickel depends on grain size because the fraction of each type of GB and the density of trapping sites for hydrogen (and therefore GB diffusivity of hydrogen) change as grain size varies in the experiment.

\section{Results for systems with multiple types of GBs}

We extend our kMC simulations to investigate $D_{\text {eff }}$ in model systems that contain multiple types of GBs. These systems resemble closely the GB networks in actual polycrystalline materials. A comprehensive investigation of the dependence of $D_{\text {eff }}$ on the properties of GB networks, such as the diffusivity ratio and the fraction of each type of GB, is beyond the scope of this work. Here, we mainly demonstrate that our model is applicable to a system with multiple GB types and show the effects of including more types of GBs on the $D_{\text {eff }}$. 
Table 1: Diffusivities of the ten types of GBs in five different cases considered in this study. $c_{v d}$ is the coefficient of variation of the diffusivity for each case (see text for details).

\begin{tabular}{c|cccccccccc|c}
\hline Case No. & $D_{1}$ & $D_{2}$ & $D_{3}$ & $D_{4}$ & $D_{5}$ & $D_{6}$ & $D_{7}$ & $D_{8}$ & $D_{9}$ & $D_{10}$ & $c_{v d}$ \\
\hline 1 & 5 & 9980 & 9985 & 9990 & 9995 & 10005 & 10010 & 10015 & 10020 & 19995 & 0.471 \\
\hline 2 & 5 & 10 & 9985 & 9990 & 9995 & 10005 & 10010 & 10015 & 19990 & 19995 & 0.667 \\
\hline 3 & 5 & 10 & 15 & 9990 & 9995 & 10005 & 10010 & 19985 & 19990 & 19995 & 0.810 \\
\hline 4 & 5 & 10 & 15 & 20 & 9995 & 10005 & 19980 & 19985 & 19990 & 19995 & 0.940 \\
\hline 5 & 5 & 10 & 15 & 20 & 25 & 19975 & 19980 & 19985 & 19990 & 19995 & 1.053 \\
\hline
\end{tabular}

As an example, we investigate a system that contains ten types of GBs, where the fraction of each type is $10 \%$. We consider 5 different cases for the GB diffusivities, as shown in Table 1. To enable a meaningful comparison, in all cases, the maximum and minimum diffusivities are the same, and so is the average diffusivity defined as $\bar{D}=\sum_{i=1}^{10} f_{i} D_{i}$, where $f_{i}$ and $D_{i}$ are the fraction and the diffusivity of the $i$-th GB, respectively. Moreover, GB diffusivities in each case can be categorized into three groups: low-diffusivity $\left(D_{i}<50\right)$, intermediate diffusivity $\left(D_{i} \approx 10^{4}\right)$, and high-diffusivity $\left(D_{i} \approx 2 \times 10^{4}\right)$. In order to make a comparison between the current simulations and the earlier simulations on systems containing two types of GBs (see Section 3), case 5 in Table 1 includes only low- and high-diffusivity GBs and therefore it is equivalent to a system with two types of GBs. The diffusivities in these 5 cases are chosen to have different diffusivity spread while keep the minimum, the maximum, and the average diffusivities the same. Here we again use the coefficient of variation $c_{v d}=\frac{\sigma}{\mu}$ to represent the diffusivity spread, where $\mu=\bar{D}$ is the mean GB diffusivity and $\sigma$ is the standard deviation of the GB diffusivity. Here the mean and standard deviations are taken over the 10 values for each case, and is not based on a KMC simulation. The coefficient of variation of the diffusivity, $c_{v d}$, therefore differs greatly in meaning from the coefficient of variation of the effective diffusivity $c_{v}$ introduced in Section 3. Since $\mu$ is the same in all cases, $c_{v d}$ is proportional to $\sigma$. It can be seen from Table 1 that $c_{v d}$ is different in the five cases, and it has the largest value in case 5 . One should note that the diffusivities in Table 1 can also be divided into two groups: one group with diffusivity on the order of 10 and the other on the order of $10^{4}$. For this reason, all 5 cases in Table 1 resemble systems with two GB types and they allow us to examine the qualitative behavior of $D_{\text {eff }}$ when multiple GB types instead of two GB types are considered.

Figure 5a shows the effective diffusivity $D_{\text {eff }}$ calculated for each case as a function of $c_{v d}$, which is the measure of the spread in diffusivity values among the ten GB types. We find that $D_{\text {eff }}$ decreases with increasing $c_{v d}$. In addition, the system with only two types of GBs (case (5)) has generally a lower effective diffusivity than a system with more GB types, provided that the maximum, the minimum, and the average diffusivities are the same. The diffusivity spread in a system with multiple GB types reaches its minimum $\left(c_{v d}=0\right)$ when the GB diffusivity is uniform (i.e., $D_{i}=\bar{D}$ ). In this case, $D_{\text {eff }}$ has its maximum value, which is $\bar{D}$. From Fig. 5 a we can also see that in a system with multiple GBs, low-diffusivity GBs have more impact on $D_{\text {eff }}$ than highdiffusivity GBs. Specifically, when the fraction of low-diffusivity GBs $f_{\mathrm{L}}$ is the same as the fraction of high-diffusivity GBs $f_{\mathrm{H}}$ (i.e., $\left.f_{\mathrm{L}}=f_{\mathrm{H}}\right), D_{\text {eff }}$ decreases as $f_{\mathrm{L}}\left(=f_{\mathrm{H}}\right)$ increases. The dominant role of low-diffusivity GBs in $D_{\text {eff }}$ is similar to systems with two types of GBs when the fraction of HEGBs is smaller than the percolation threshold (see Fig. 2).

For the sake of further comparison between the behavior of $D_{\text {eff }}$ in systems with two and more 
than two types of GBs, we also examine the EMT prediction of $D_{\text {eff }}$, the grain size effect, and fluctuation of $D_{\text {eff }}$. Figure $5 \mathrm{~b}$ gives the relative difference in $D_{\text {eff }}$ obtained from kMC and EMT

(i.e., $\left.\left(D_{\text {eff }}^{\mathrm{kMC}}-D_{\text {eff }}^{\mathrm{EMT}}\right) / D_{\text {eff }}^{\mathrm{EMT}}\right)$ as a function of the coefficient of variation $c_{v d}$. It shows that the deviation of EMT prediction from $\mathrm{kMC}$ results increases as $c_{v d}$ (i.e., the spread in diffusivity) increases. Since the physical meaning of the spread in diffusivity is the same in a system with multiple GB types and in a system with only two GB types, it is not surprising that in a system with multiple GB types, EMT works better for samples with smaller $c_{v d}$. The dependence of $D_{\text {eff }}$ on the average grain size in the five cases studied here is shown in Fig. 5c. It can be seen that, consistently with what we found in a system with two types of GBs, $D_{\text {eff }}$ in systems with multiple GB types does not depend on the grain size as long as only GB diffusion is activated. Including more types of GBs not only does not induce a grain size dependence of $D_{\text {eff }}$, but also does not have a strong effect on the fluctuation of $D_{\text {eff }}$, as shown in Fig. 5d. We find that although the coefficient of variation of $D_{\text {eff }}$ in systems with multiple GB types increases slightly as the diffusivity spread increases (from case 1 to case 5), the values of $c_{v}$ is similar in systems with two and multiple GB types. Consistently with the trend found in systems with two GB types (see Figures 4b and 4c), the fluctuation of $D_{\text {eff }}$ decreases as the system size increases in a system with multiple GB types, and more importantly, the fluctuation can be significant even if a system has more than $10^{3}$ grains. The latter again demonstrates that the details of microstructure should be taken into account when the macroscopic diffusivity is measured in a specific polycrystalline sample.

In summary, extending the model systems to include more than two GB types does not have an impact on the existence or lack of grain size effects on diffusion and on fluctuation of diffusion coefficients among randomly generated microstructures with the same macroscopic parameters. When the minimum, the maximum and the average diffusivities of individual GBs are kept constant, including more than two GB types leads to a faster diffusion. For materials with multiple types of GBs, low-diffusivity GBs have more impact on $D_{\text {eff }}$ in the sense that when the fractions of lowdiffusivity GBs and high-diffusivity GBs are kept the same (e.g., cases 1-5 in Table 1), $D_{\text {eff }}$ decreases as these fractions increase (e.g., from case 1 to case 5 in Table 1). We also find that the EMT model captures the diffusion behavior better in systems with a smaller spread in diffusivity values among the different GB types. Finally, one should note that the cases studied here are special because all ten GB types are equally probable in the sample, which may not reflect the complexity in actual GB structure. Nevertheless, the properties of $D_{\text {eff }}$ shown above do not depend on the number of GB types or their fraction, and therefore they are expected to provide a general behavior of $D_{\text {eff }}$ in realistic GB systems with multiple GB characters.

\section{Conclusions}

We have developed a kinetic Monte Carlo model to evaluate the effective diffusivity in GB networks in polycrystalline materials. This model connects the atomic-level information of hopping processes with the coarse-grained diffusion on GB planes, which allows us to conduct macroscopic simulations without resolving atomic details. Several features of the effective diffusivity have been found from the numerical experiments. First, in the kinetic limit when GB diffusion is the dominant transport mechanism, the effective diffusivity through a polycrystalline sample does not depend on the grain size. Secondly, the dimensionality and the type of GB networks mainly affect the percolation threshold and the critical exponents in the percolation theory, and they do not change the qualitative dependence of the effective diffusivity on the diffusivity contrast and the fraction of GB types. We also find that the effective diffusivity exhibits fluctuations due to the statistical 
nature of GB distribution. In addition, our results show that EMT approximates the effective diffusivity with a reasonable accuracy not only in low-diffusivity contrast systems, but also in high-diffusivity contrast systems when the fraction of HEGBs is significantly different from the percolation threshold. In all cases, GEM can be used to predict the effective diffusivity if the percolation threshold and the critical exponents are known. Finally, including more types of GBs generally increases the effective diffusivity, provided that the maximum, the minimum and the average diffusivities, are kept the same.

Although the properties of the effective diffusivity shown above are based on simplified GB systems, these properties do not depend on specific materials or GB characters, and they shed light on the qualitative behavior of the effective diffusivity in systems containing two or more GB types. The presented modeling framework is flexible so that it will be straightforward to include additional effects such as the correlation of GB types. This model can be easily applied to specific materials systems with complex GB networks, realistic diffusivities, and with a variable fraction of each GB type.

\section{Acknowledgement}

This research is being performed using funding received from the DOE Office of Nuclear Energy's Nuclear Energy University Programs contract number 00089350.

\section{References}

[1] I. Kaur, Y. Mishin, W. Gust, Fundamentals of Grain and Interphase Boundary Diffusion, Wiley, Chichester, UK, 1995.

[2] Y. Mishin, C. Herzig, Grain boundary diffusion: Recent progress and future research, Mat. Sci. Eng. A 260 (1999) 55.

[3] J. Fisher, Calculation of Diffusion Penetration Curves for Surface and Grain Boundary Diffusion, J. Appl. Phys. 22 (1951) 74.

[4] L. Harrison, Influence of dislocations on diffusion kinetics in solids with particular reference to the alkali halides, Trans. Faraday Soc. 57 (1961) 1191.

[5] R. Whipple, Concentration contours in grain boundary diffusion, Philos. Mag. 45 (1954) 1225.

[6] A. Le Claire, The analysis of grain boundary diffusion measurements, Brit. J. Appl. Phys. 14 (1963) 351.

[7] T. Suzuoka, Lattice and grain boundary diffusion in polycrystals, Trans. Jpn. Inst. Metals 2 (1961) 25.

[8] G. Gilmer, H. Farrel, Grain boundary diffusion in thin films: I. The isolated grain boundary, J. Appl. Phys. 47 (1976) 3792.

[9] G. Gilmer, H. Farrel, Grain boundary diffusion in thin films: II. Multiple grain boundaries and surface diffusion, J. Appl. Phys. 47 (1976) 4373.

[10] W. Preis, W. Sitte, Grain boundary diffusion through thin films. Application to permeable surfaces, J. Appl. Phys. 79 (1996) 2986. 
[11] W. Preis, W. Sitte, Fast grain boundary diffusion and rate-limiting surface exchange reactions in polycrystalline materials, J. Appl. Phys. 97 (2005) 093504.

[12] D. Turnbull, R. Hoffman, The effect of relative crystal and boundary orientations on grain boundary diffusion rates, Acta Metall. 2 (1954) 419.

[13] R. Hoffman, Anisotropy of grain boundary self-diffusion, Acta Metall. 4 (1956) 97.

[14] B. Straumal, B. Bokshtein, L. Klinger, L. Shvindlerman, Indium diffusion along interphase twist boundaries SnGe, Scripta Metall. 15 (1981) 1197.

[15] J. Sommer, C. Herzig, S. Mayer, W. Gust, Grain boundary self-diffusion in silver bicrystals, Defect Diff. Forum 66-69 (1989) 843.

[16] E. Budke, C. Herzig, S. Prokofjev, L. Shvindlerman, Orientation dependence of Au and Cu Diffusion Along Symmetric [001] Tilt Grain Boundaries in Cu, Mater. Sci. Forum 207-209 (1996) 465.

[17] Q. Ma, R. Balluffi, Diffusion along [001] tilt boundaries in the Au/Ag system I. Experimental result, Acta Metall. Mater. 41 (1993) 133.

[18] A. Suzuki, Y. Mishin, Atomic mechanisms of grain boundary diffusion: Low versus high temperatures, J. Mat. Sci. 40 (2005) 3155.

[19] L. Lim, T. Watanabe, Fracture toughness and brittle-ductile transition controlled by grain boundary character distribution (GBCD) in polycrystals, Acta Metall. Mater. 38 (1990) 2507.

[20] V. Gertsman, K. Tangri, R. Valiev, On the grain boundary statistics in metals and alloys susceptible to annealing twinning, Acta Metall. Mater. 42 (1994) 1785.

[21] L. Fionova, Grain boundary ensembles in materials with f.c.c., b.c.c. and diamond structures, Mater. Chem. Phys. 37 (1994) 201.

[22] T. Watanabe, The impact of grain boundary character distribution on fracture in polycrystals, Mat. Sci. Eng. A 176 (1994) 39.

[23] V. Gertsman, M. Janecek, K. Tangri, Grain boundary ensembles in polycrystals, Acta Mater. 44 (1996) 2869.

[24] W. Hart, On the role of dislocations in bulk diffusion, Acta Metallogr. 5 (1957) 597.

[25] A. Mortlock, The effect of segregation on the solute diffusion enhancement due to the presence of dislocations, Acta Metallogr. 8 (1960) 132.

[26] J. Kalnins, E. Kotomin, J. Maier, Calculations of the effective diffusion coefficient for inhomogeneous media, J. Phys. Chem. Solids 63 (2002) 449.

[27] I. Belova, G. Murch, Diffusion in nanocrystalline materials, J. Phys. Chem. Solids 64 (2003) 873.

[28] I. Belova, G. Murch, The effective diffusivity in polycrystalline material in the presence of interphase boundaries, Philog. Mag. 84 (2004) 17. 
[29] I. Belova, G. Murch, Calculation of the effective conductivity and diffusivity in composite solid electrolytes, J. Phys. Chem. Solids 66 (2005) 722.

[30] Y. Chen, C. Schuh, Geometric considerations for diffusion in polycrystalline solids, J. Appl. Phys. 101 (2007) 063524.

[31] D. Shrader, S. Khalil, T. Gerczak, T. Allen, A. Heim, I. Szlufarska, D. Morgan, Ag diffusion in cubic silicon carbide, J. Nuc. Mat. 408 (2011) 257.

[32] S. Khalil, N. Swaminathan, D. Shrader, A. Heim, D. Morgan, I. Szlufarska, Diffusion of Ag along $\Sigma 3$ grain boundaries in 3C-SiC, Phys. Rev. B 84 (2011) 214104.

[33] Y. Chen, C. Schuh, Diffusion on grain boundary networks: Percolation theory and effective medium approximations, Acta Mater. 54 (2006) 4709.

[34] A. Suzuki, Y. Mishin, Atomistic Modeling of Point Defects and Diffusion in Copper Grain Boundaries, Interface Sci. 11 (2003) 131.

[35] J. Harding, D. Harris, Simulation of grain-boundary diffusion in ceramics by kinetic Monte Carlo, Phys. Rev. B 63 (2001) 094102.

[36] A. Pedersen, H. Jonsson, Simulations of hydrogen diffusion at grain boundaries in aluminum, Acta Mater. 57 (2009) 4036.

[37] Y. Du, J. Rogal, R. Drautz, Diffusion of hydrogen within idealized grains of bcc Fe: A kinetic Monte Carlo study, Phys. Rev. B 86 (2012) 174110.

[38] R. Quey, P. Dawson and F. Barbe, Large-scale 3D random polycrystals for the finite element method: Generation, meshing and remeshing, Comp. Met. Appl. Mech. Eng. 200 (2011) 17291745 .

[39] M. Frary, C. Schuh, Nonrandom percolation behavior of grain boundary networks in high-Tc superconductors, App. Phys. Lett. 83 (2003) 3755.

[40] M. Frary, C. Schuh, Percolation and statistical properties of low- and high-angle interface networks in polycrystalline ensembles, Phys. Rev. B 69 (2004) 134115.

[41] M. Frary, C. Schuh, Connectivity and percolation behaviour of grain boundary networks in three dimensions, Philo. Mag. 85 (2005) 1123.

[42] B. Bokstein, V. Ivanov, O. Oreshina, A. Peteline, S. Peteline, Direct experimental observation of accelerated Zn diffusion along triple junctions in Al, Mater. Sci. Eng. A 302 (2001) 151.

[43] T. Frolov, Y. Mishin, Molecular dynamics modeling of self-diffusion along a triple junction, Phys. Rev. B 79 (2009) 174110.

[44] T. Angsten, T. Mayeshiba, H. Wu, and D. Morgan, Elemental vacancy diffusion database from high-throughput first-principles calculations for fcc and hcp structures, New J. Phys. 16 (2014) 015018 .

[45] https://materialshub.org/resources/diffcalc 
[46] P. Millett, M. Tonks, S. Biner, Grain boundary percolation modeling of fission gas release in oxide fuels, J. Nuc. Mat. 424 (2012) 176.

[47] A. Becker, R. Ziff, Percolation thresholds on two-dimensional Voronoi networks and Delaunay triangulations, Phys. Rev. E 80 (2009) 041101.

[48] V. Vyssotsky, S. Gordon, H. Frisch, J. Hammersley, Critical Percolation Probabilities (Bond Problem), Phys. Rev. 123 (1961) 1566.

[49] A. Hunt, Percolation Theory for Flow in Porous Media, Springer, Berlin, 2005.

[50] S. Kirkpatrick, Percolation and Conduction, Rev. Mod. Phys. 45 (1973) 574.

[51] D. Stauffer, A. Aharony, Introduction to Percolation Theory, CRC Press, Philadelphia, USA, 1994.

[52] M. Isichenko, Percolation, statistical topography, and transport in random media, Rev. Mod. Phys. 64 (1992) 961.

[53] J. Clerc, G. Giraud, J. Laugier, J. Luck, The electrical conductivity of binary disordered systems, percolation clusters, fractals and related models, Adv. Phys. 39 (1990) 191.

[54] P. Li, W. Strieder, Critical exponents for conduction in a honeycomb random site lattice, J. Phys. C 15 (1982) L1235.

[55] M. Sahimi, On the relationship between the critical exponents of percolation conductivity and static exponents of percolation, J. Phys. A 17 (1984) L601.

[56] J. Straley, Critical exponents for the conductivity of random resistor lattices, Phys. Rev. B 15 (1977) 5733.

[57] I. Webman, J. Jortner, M. Cohen, Critical exponents for percolation conductivity in resistor networks, Phys. Rev. B 16 (1977) 2593.

[58] M. Sahimi, B. Hughes, L. Scriven, H. Davis, J. Phys. C 16 (1983) L521.

[59] D. McLachlan, An equation for the conductivity of binary mixtures with anisotropic grain structures, J. Phys. C 20 (1987) 865.

[60] D. McLanchlan, M. Blaskiewicz, R. Newnham, Electrical Resistivity of Composites, J. Am. Ceram. Soc. 73 (1990) 2187.

[61] J. Wu, D. McLachlan, Percolation exponents and thresholds obtained from the nearly ideal continuum percolation system graphite-boron nitride, Phys. Rev. B 56 (1997) 1236.

[62] H. Berg, Random Walks in Biology, Princeton University Press, Princeton, NJ, 1993.

[63] A. Oudriss, J. Creus, J. Bouhattate, E. Conforto, C. Berziou, C. Savall, X. Feaugas, Grain size and grain-boundary effects on diffusion and trapping of hydrogen in pure nickel, Acta Mater. 60 (2012) 6814. 


\section{Figure Caption:}

Figure 1 (Color online) (a) A typical 3D Voronoi GB network with 2000 grains, where the triangular mesh is generated in each GB. (b) Average fraction of percolating HEGBs as a function of the fraction $f_{\mathrm{H}}$ of HEGBs in $2 \mathrm{D}$ and 3D Voronoi GB networks. (c) The effective diffusivity as a function of mesh density with different diffusivity ratios $\frac{D_{\mathrm{H}}}{D_{\mathrm{L}}}$ when fraction $f_{\mathrm{H}}$ of HEGBs is 0.5 and the diffusivity $D_{\mathrm{L}}$ in LEGB is 1.0. In (b) and (c), the error bar is within the thickness of the symbols used in the points.

Figure 2 (Color online) Dependence of the effective diffusivity $D_{\text {eff }}$ on $\frac{D_{\mathrm{H}}}{D_{\mathrm{L}}}$ for different fractions $f_{\mathrm{H}}$ of HEGBs. The solid lines and dashed lines correspond to the cases when $f_{\mathrm{H}}$ is smaller and larger than the percolation threshold $f_{\mathrm{p}}$, respectively.

Figure 3 (Color online) (a) Dependence of $D_{\text {eff }}$ on $f_{\mathrm{H}}$ for different ratios $\frac{D_{\mathrm{H}}}{D_{\mathrm{L}}}$. Symbols, dash lines and solid lines correspond to the results from kMC simulations, predictions of the EMT theory, and predictions of the GEM theory with $f_{\mathrm{p}}=0.18, t=1.30$, and $s=1.22$. (b) The ratio of $D_{\text {eff }}$ values calculated from $\mathrm{kMC}$ and from EMT for different values of $\frac{D_{\mathrm{H}}}{D_{\mathrm{L}}}$. $D_{\text {eff }}$ as a function of (c) $f_{\mathrm{H}}-f_{\mathrm{p}}$ when $f_{\mathrm{H}}>f_{\mathrm{p}}$ and $(\mathrm{d}) f_{\mathrm{p}}-f_{\mathrm{H}}$ when $f_{\mathrm{H}}<f_{\mathrm{p}}$ calculated for different values of $\frac{D_{\mathrm{H}}}{D_{\mathrm{L}}}$. In (c) and (d), the solid lines are the curve fitting using the power-law functions described in text.

Figure 4 (Color online) (a) $\left\langle D_{\text {eff }}\right\rangle$ with error bar (estimated by the standard deviation divided by the mean) as a function of the system size when $\frac{D_{H}}{D_{L}}=1000$ and $f_{\mathrm{H}}=0.5$. Coefficient of variation of $D_{\text {eff }}$ as a function of the system size for (b) different values of $\frac{D_{\mathrm{H}}}{D_{\mathrm{L}}}$ when $f_{\mathrm{H}}=0.5$ and (c) different values of $f_{\mathrm{H}}$ when $\frac{D_{H}}{D_{L}}=100$.

Figure 5 (Color online) Analysis of a system with more than two types of GB. (a) Effective diffusivity

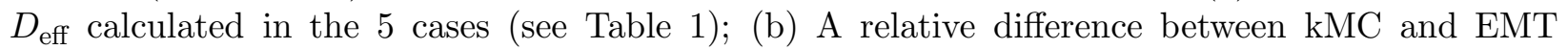
predictions as a function of the coefficient of variation of GB diffusivity; (c) Dependence of $D_{\text {eff }}$ on the normalized grain size (ratio of the average grain size to the domain size); (d) Coefficient of variation of $D_{\text {eff }}$ as a function of the system size. 


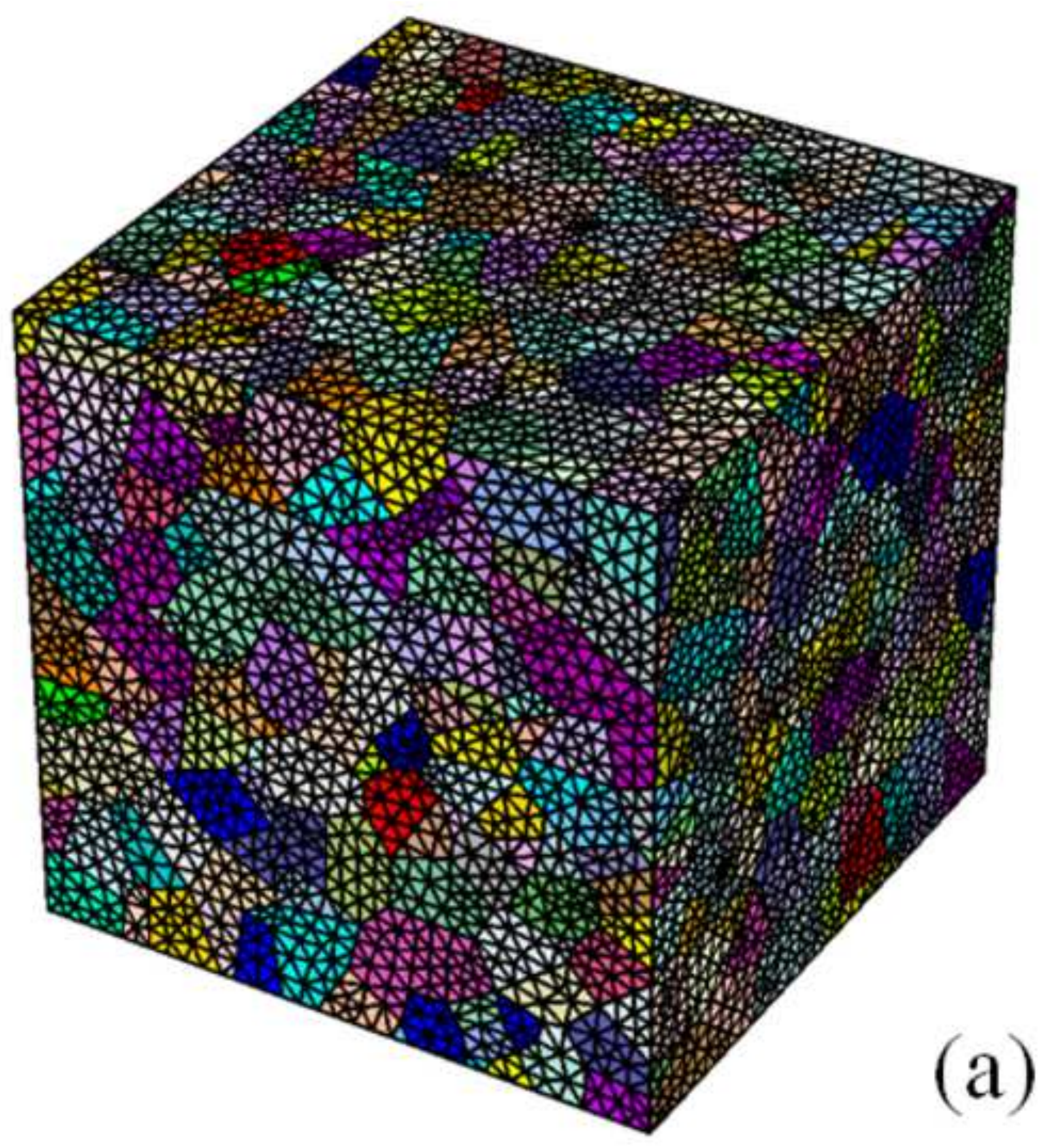




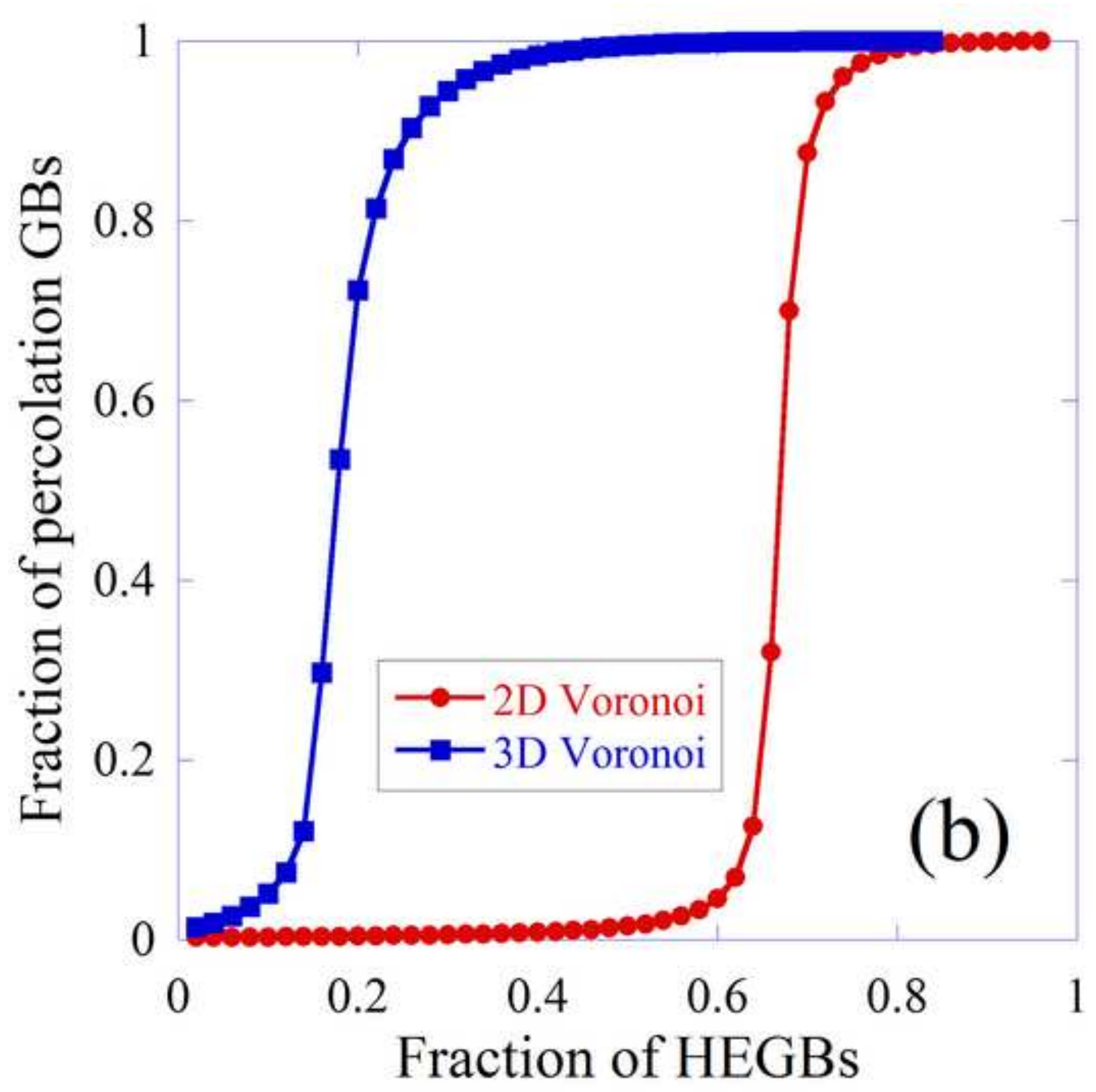




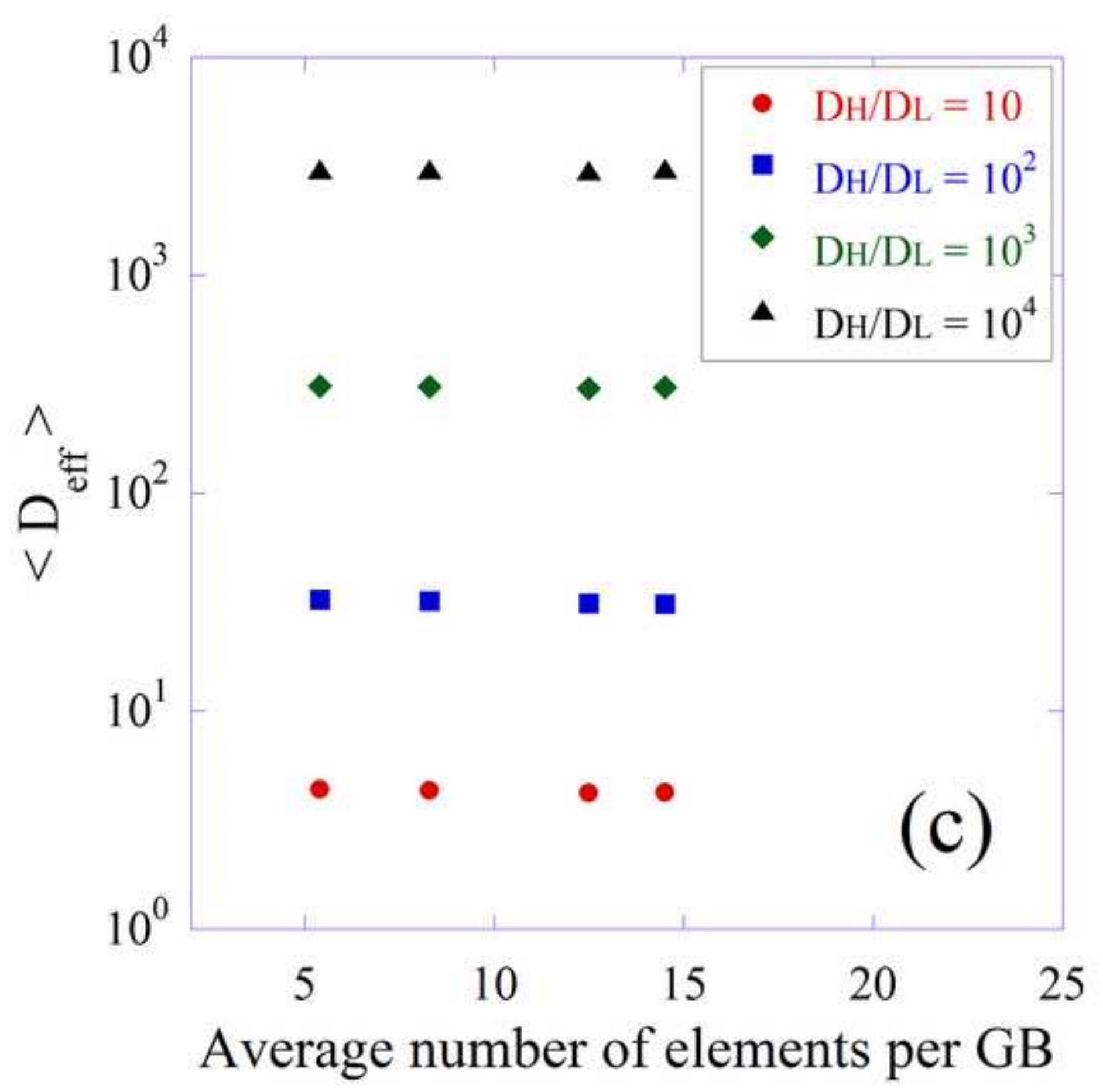




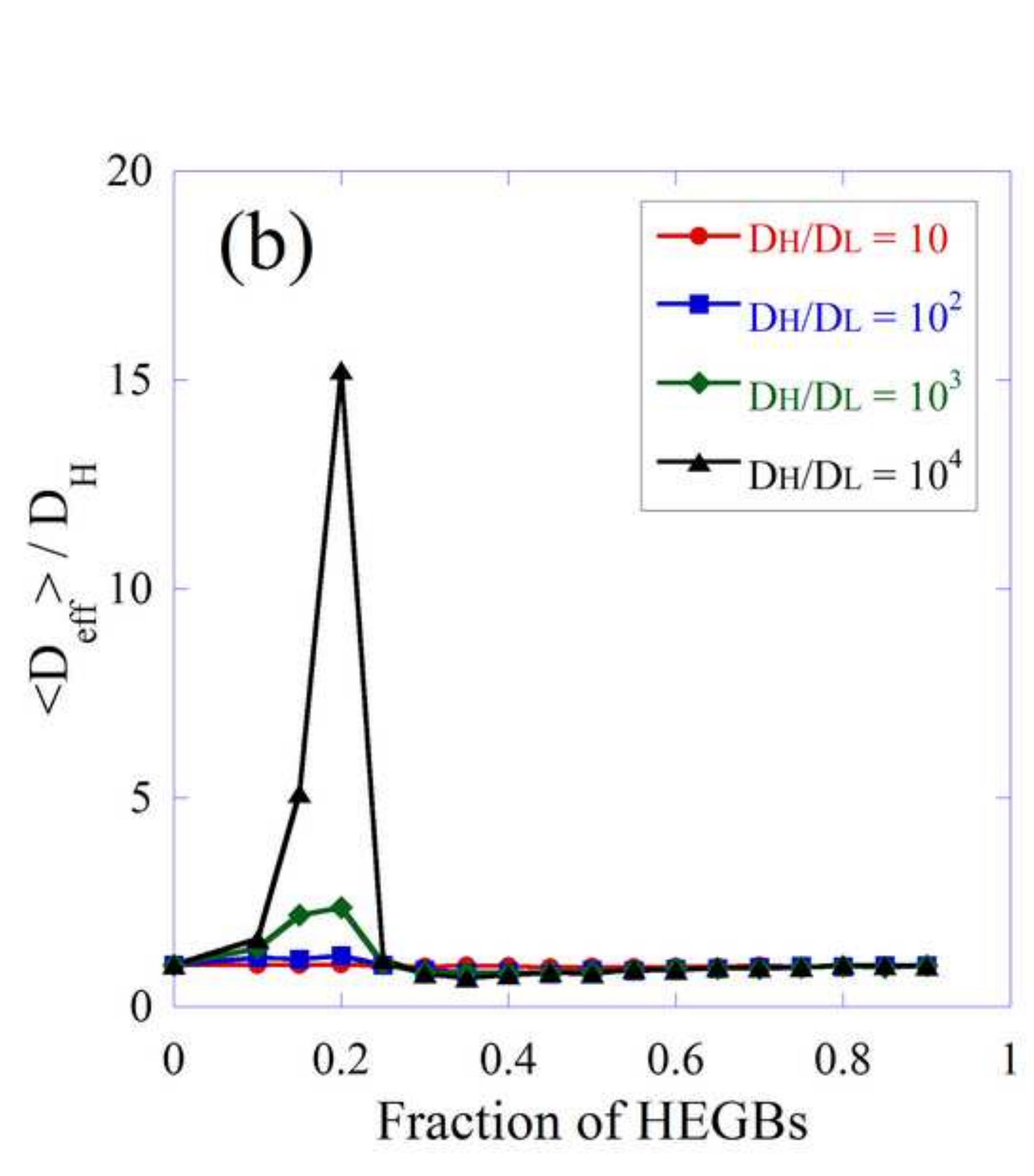




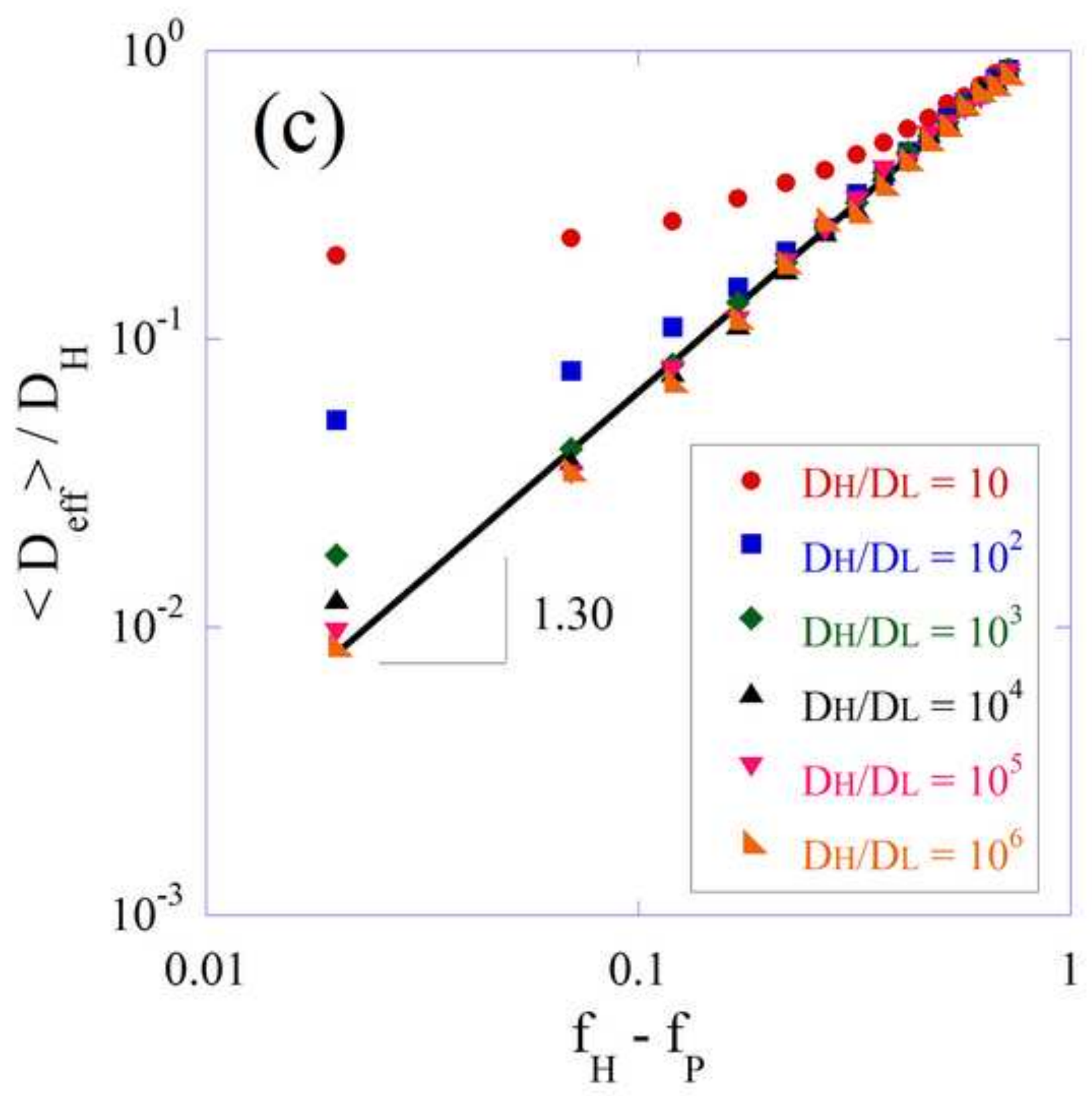

$$
\mathrm{f}_{\mathrm{H}}^{0.1}-\mathrm{f}_{\mathrm{P}}
$$



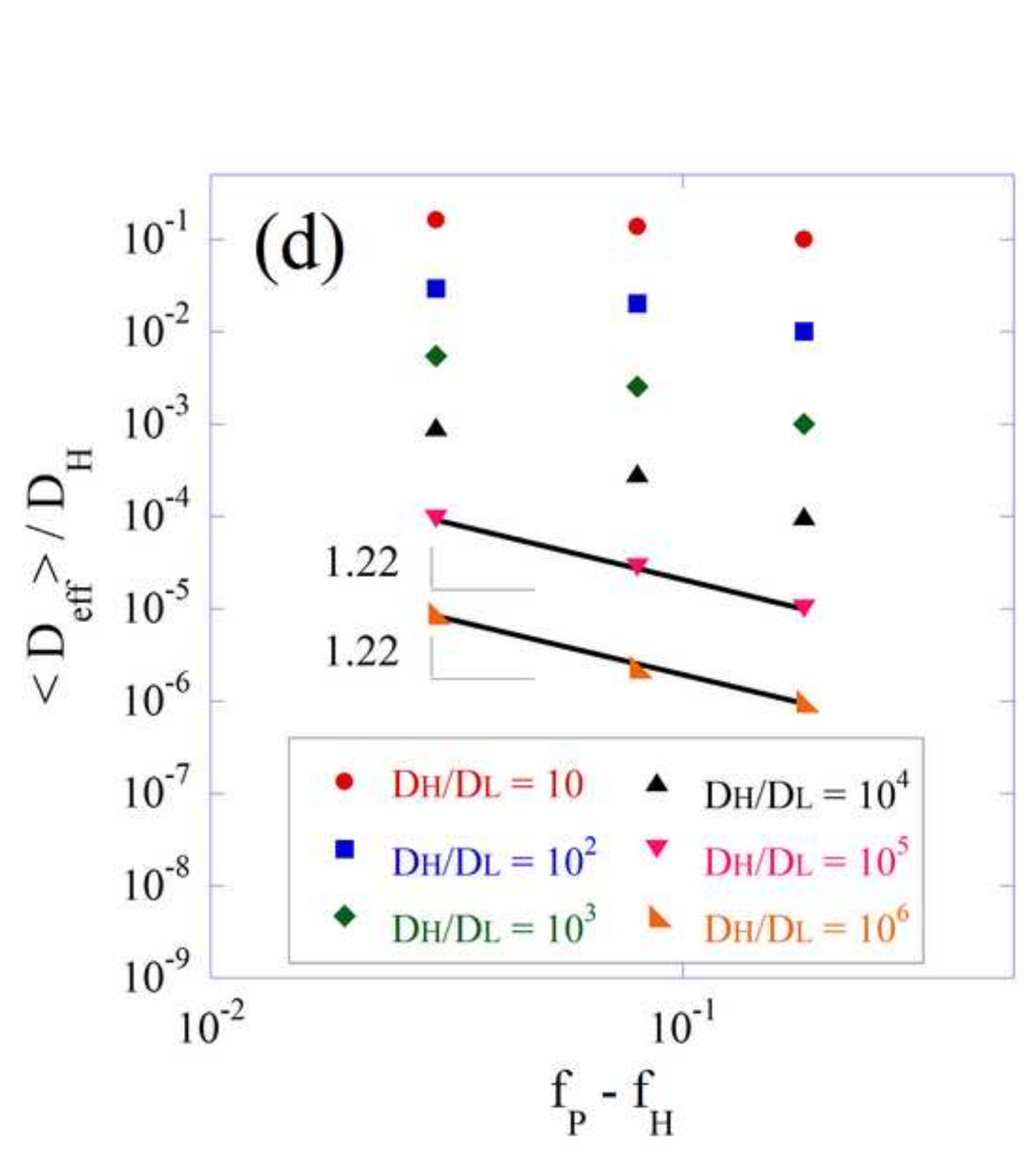


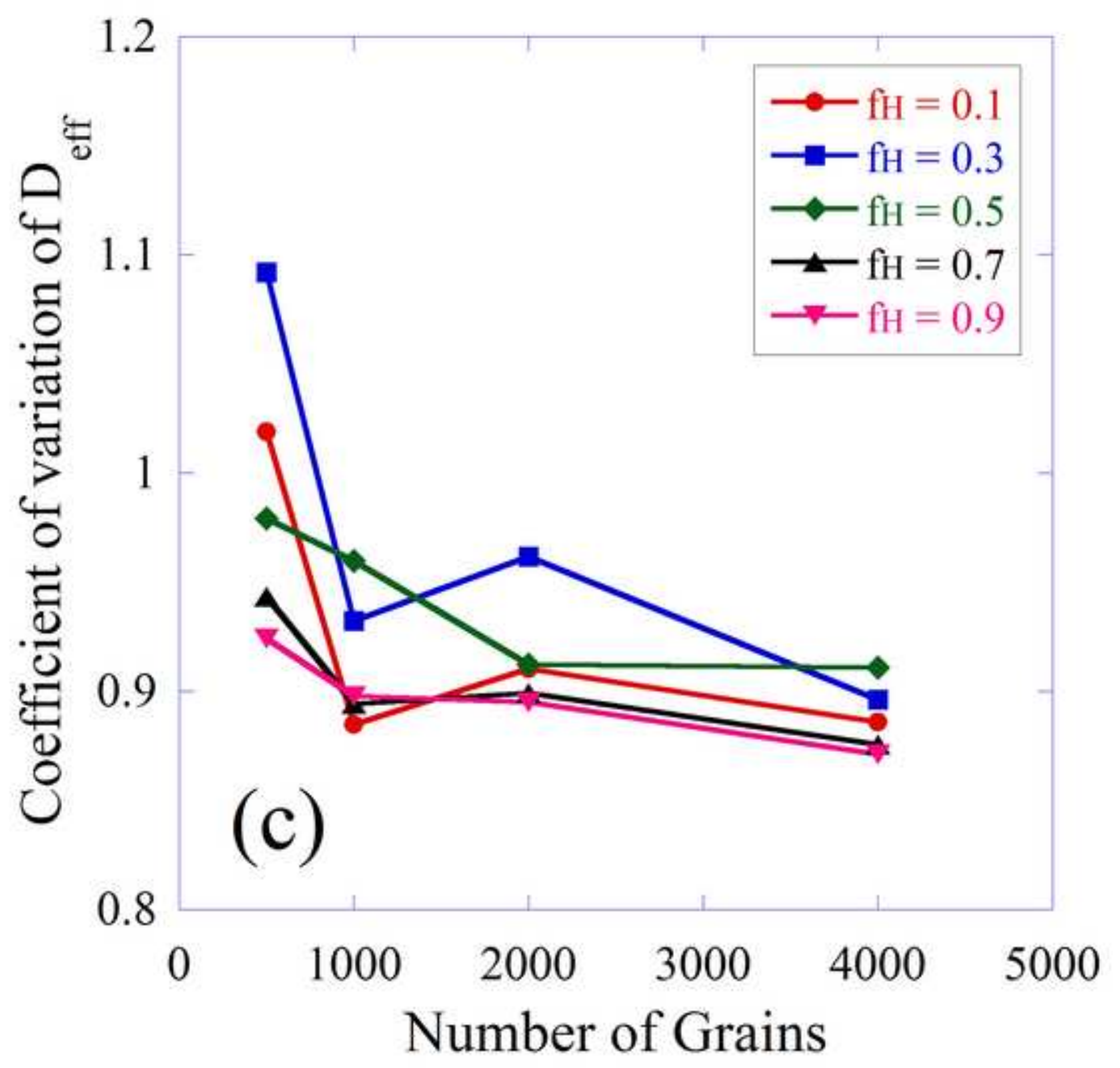




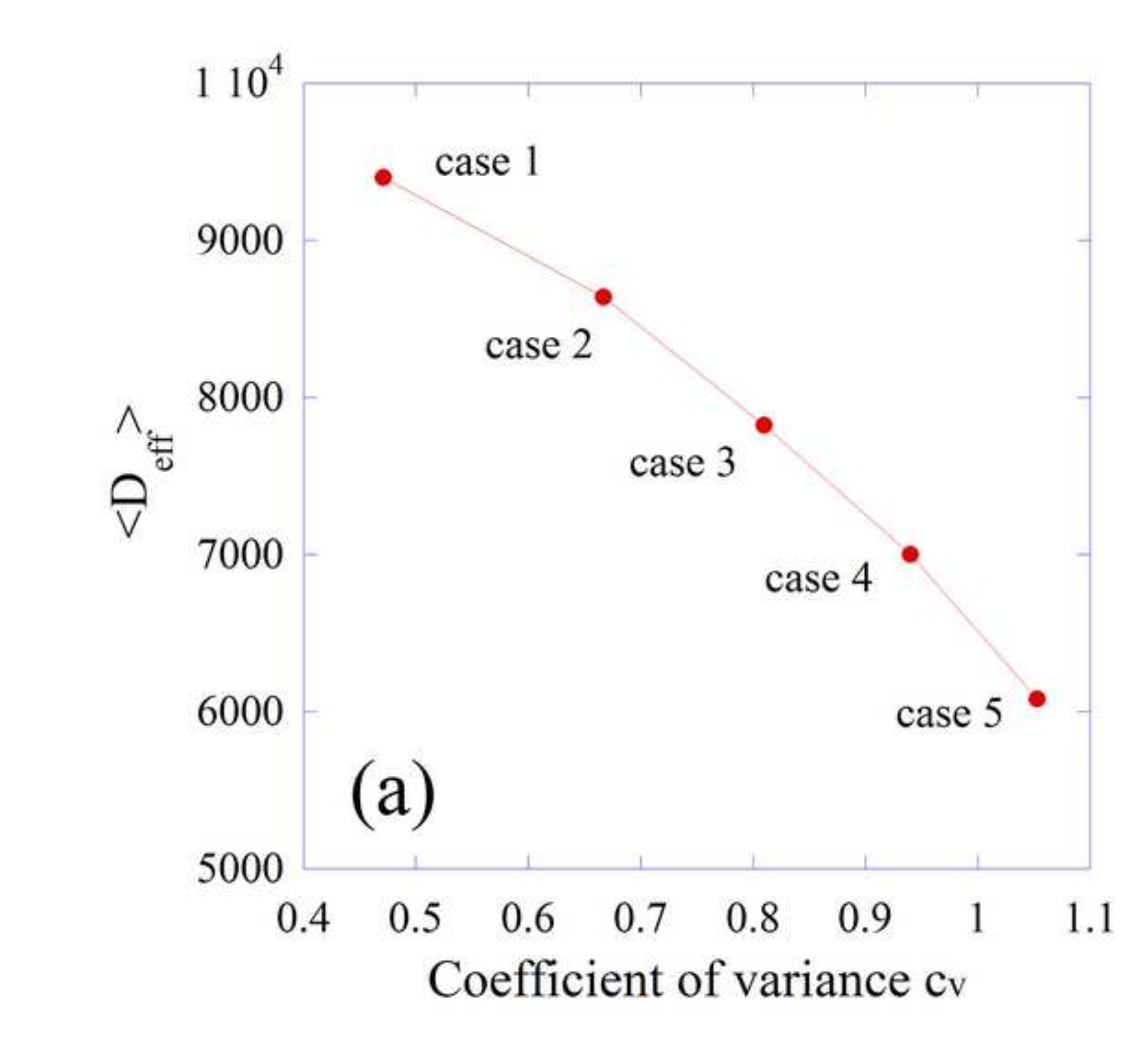

\section{Coefficient of variance $\mathrm{c} v$}

.
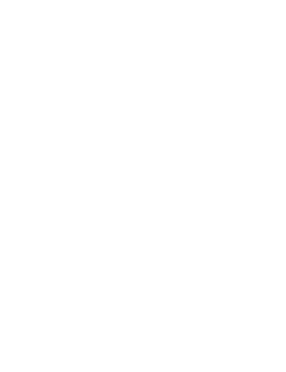

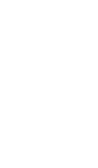
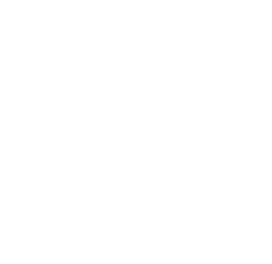

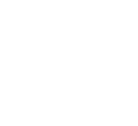




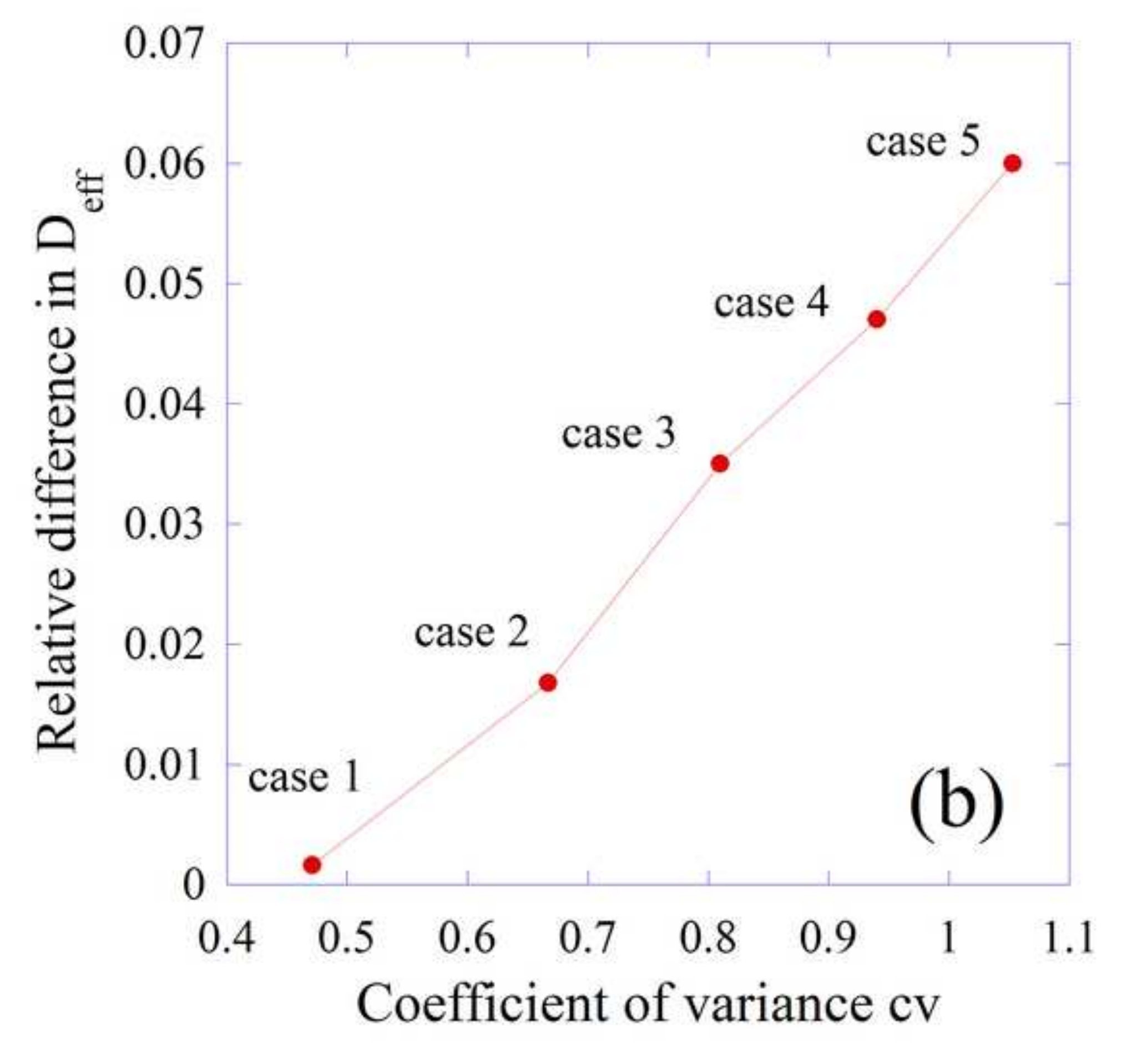

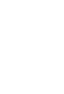
(n)

政
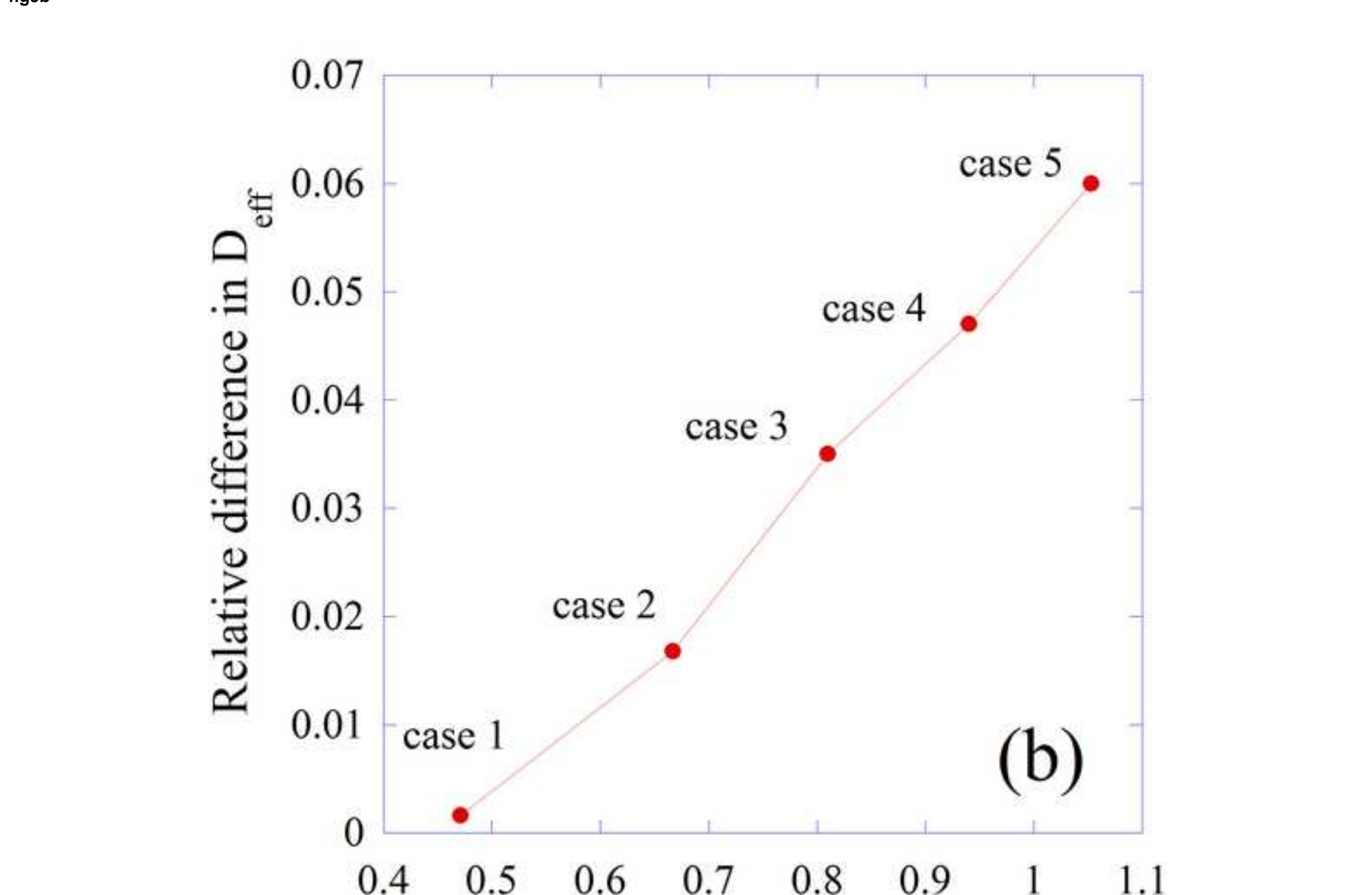

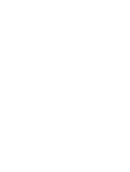
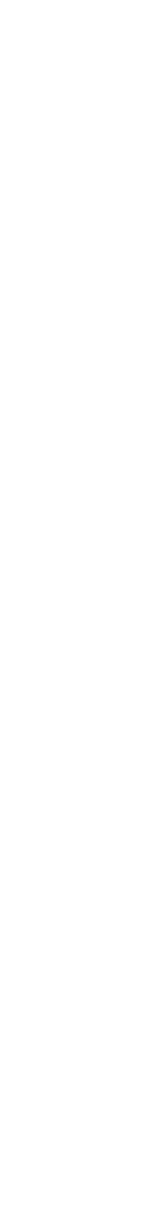$$
0.4
$$
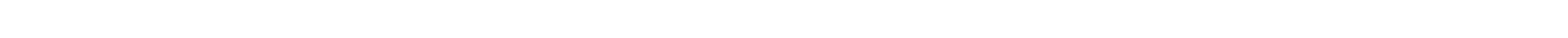


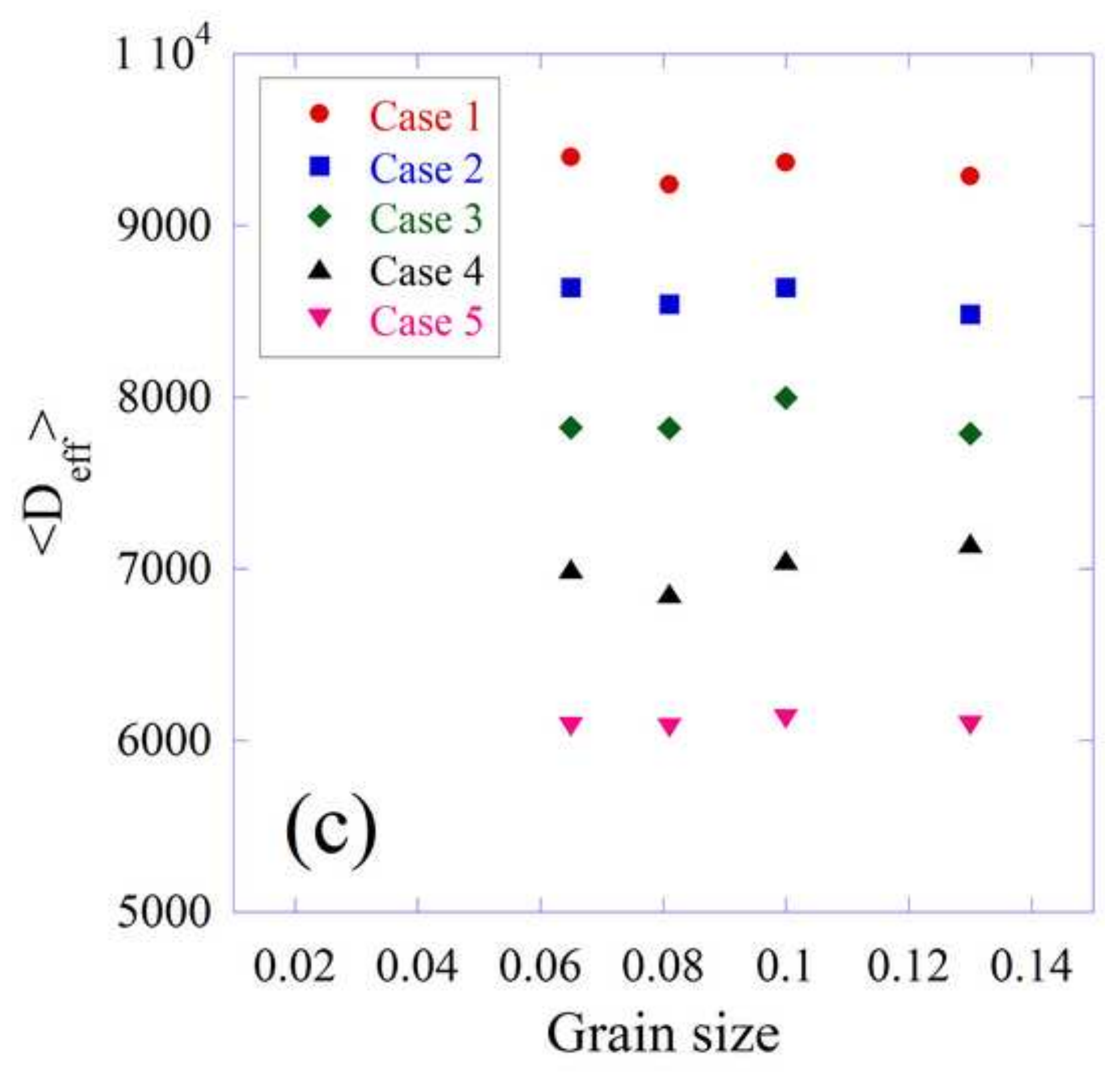

Grain size

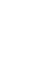

(n)




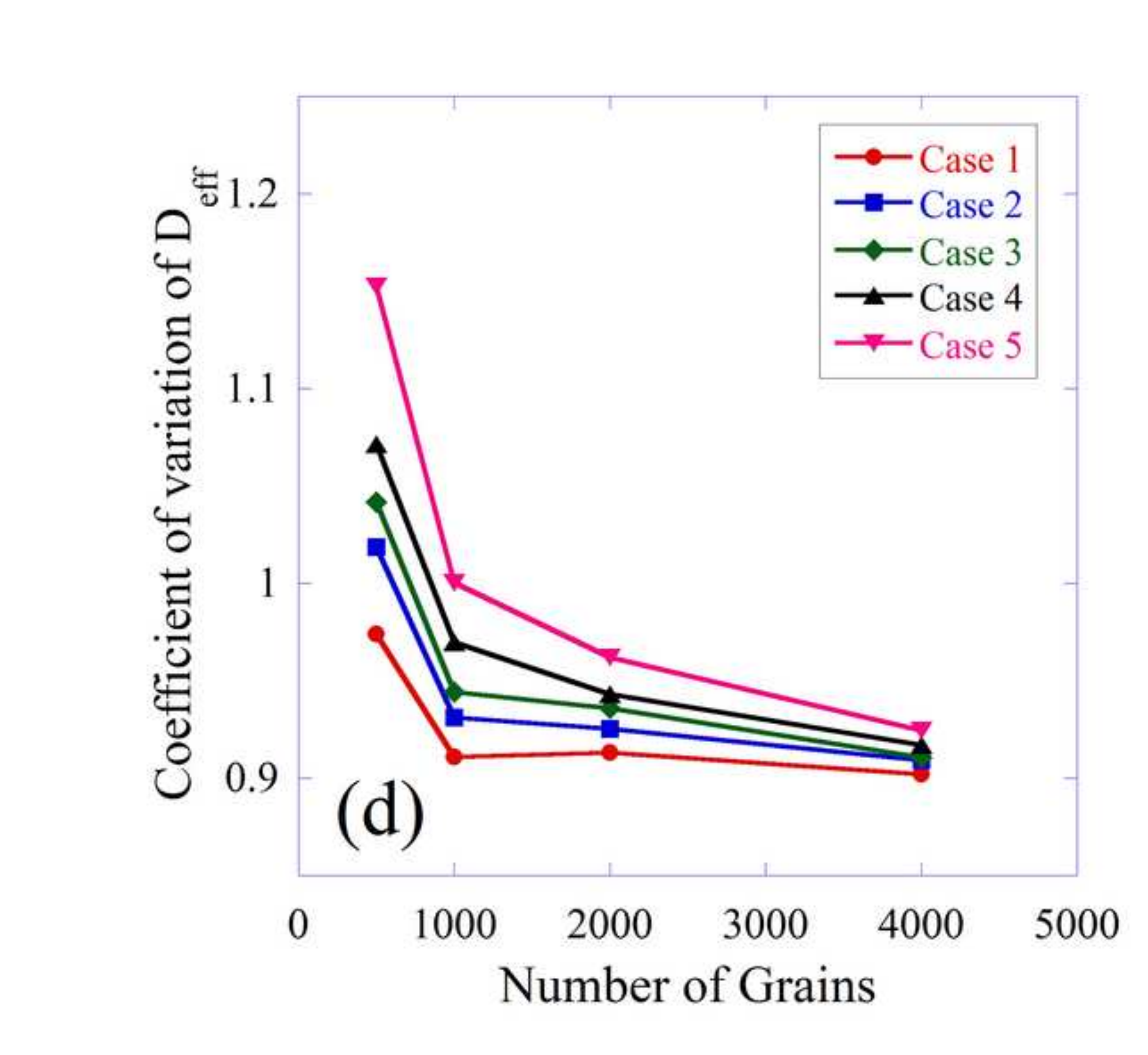

.
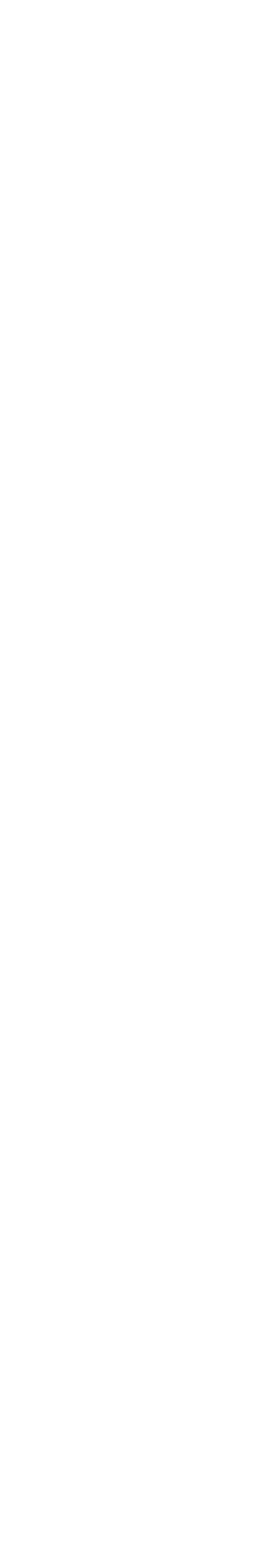

.

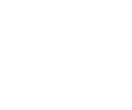

(2)

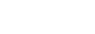


Three-dimensional grain boundary network with triangular mesh in each grain boundary plane

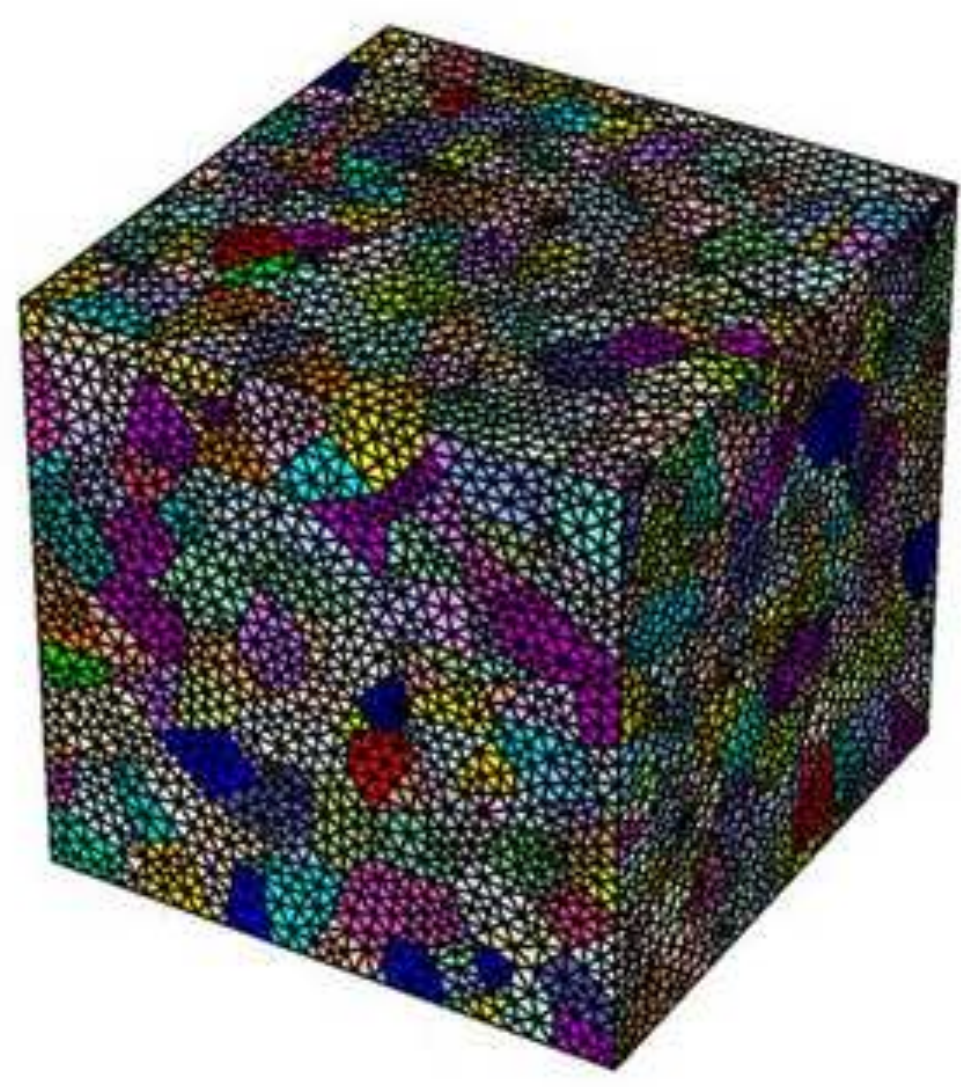

Effective diffusivity as a function of high-energy grain boundary fraction with different diffusivity ratios

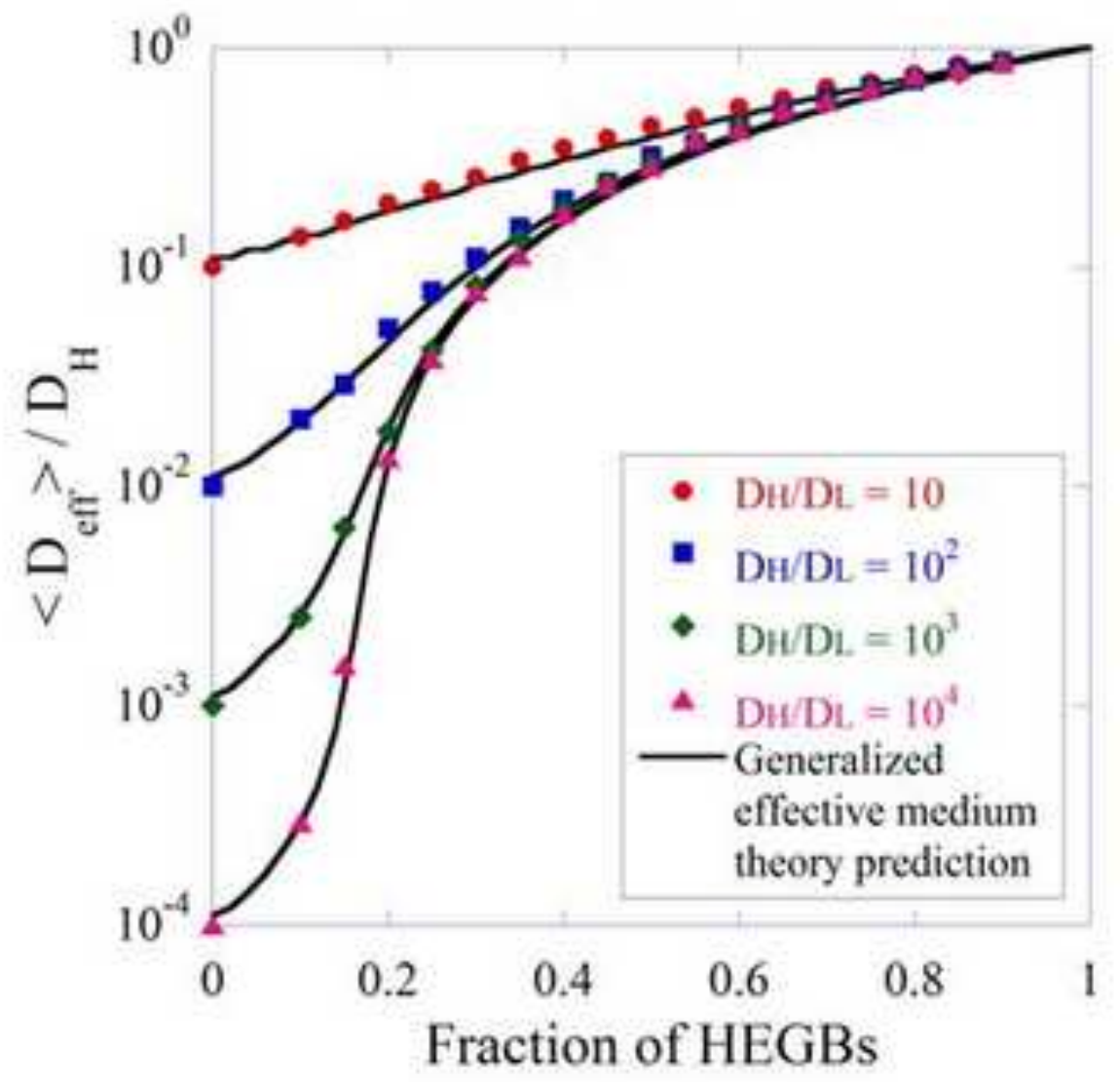

